O Teorema de Poincaré-Bendixson para campos vetoriais contínuos na garrafa de Klein 



\title{
O Teorema de Poincaré-Bendixson para campos vetoriais contínuos na garrafa de Klein ${ }^{1}$
}

\author{
Daniela Paula Demuner
}

Orientadora: Profa. Dra. Márcia Cristina Anderson Braz Federson

Orientador (in memorian): Prof. Dr. Carlos Teobaldo Gutierrez Vidalon

Tese apresentada ao Instituto de Ciências Matemáticas e de Computação da Universidade de São Paulo, como parte dos requisitos para obtenção do título de Doutor em Ciências Área: Matemática.

\section{USP - São Carlos \\ Dezembro/2008}

\footnotetext{
${ }^{1}$ Este trabalho teve suporte financeiro do $\mathrm{CNPq}$
} 

À minha família $e$ ao professor Carlos Gutierrez (in memorian). 



\section{Agradecimentos}

Agradeço acima de tudo a Deus, pelo dom da vida e por estar constantemente me provando que é possível crescer diante das dificuldades e também pela felicidade de poder compartilhar com todos a conquista de mais essa etapa importante em minha vida.

Ao professor Carlos Gutierrez, pela orientação e tempo dedicado a este trabalho.

À professora Márcia Federson, pelos ensinamentos e colaboração, que foram fundamentais para o término deste trabalho. Minha eterna gratidão!

A toda minha família, sobretudo a meus pais Benito e Laudir, que confiaram em mim e estiveram sempre ao meu lado me incentivando.

Ao meu namorado e amigo Alancardek, pelo carinho e compreensão dedicados a mim nesses anos que passei longe. Saiba que a saudade foi constante e meu amor por você só se tornou mais forte.

Aos amigos que encontrei em minha caminhada, os quais tanto me incentivaram e me ajudaram a transpor todos os obstáculos até aqui. Em especial, agradeço ao Sadao e aos amigos da pós-graduação, pela amizade, companherismo e grande apoio nos momentos difíceis.

À todos os professores e funcionários do ICMC, que de muitas formas contribuíram para a realização deste trabalho, em especial aos professores Carlos Biasi e Oziride (Didi).

Aos professores do departamento de matemática da UFES, pelo apoio e incentivo.

Ao $\mathrm{CNPq}$, pelo apoio financeiro para realização deste trabalho.

Enfim, a todos aqueles que colaboraram de alguma forma para a realização deste trabalho.

Muito Obrigada! 



\section{Resumo}

O objetivo deste trabalho é apresentar uma versão do Teorema de Poincaré-Bendixson para campos vetoriais contínuos na garrafa de Klein $K^{2}$. Como conseqüência, mostraremos que $K^{2}$ não possui campo vetorial contínuo com trajetória injetiva $\omega$-recorrente.

Além do objetivo acima, procuramos produzir um texto que compreendesse a teoria qualitativa fundamental das equações diferenciais ordinárias para campos vetoriais contínuos em variedades. Tal teoria não podia ser encontrada num único texto como este. De fato, somente poucos resultados são conhecidos para campos vetoriais contínuos em variedades. Portanto, a nossa contribuição não está somente na prova de uma versão do Teorema de Poincaré-Bendixson para $K^{2}$, mas também no desenvolvimento da teoria necessária para isto. 



\section{Abstract}

The aim of this work is to present a version of the Poincaré-Bendixson Theorem for continuous vector fields on the Klein bottle $K^{2}$. As a consequence, we prove that $K^{2}$ does not admit a continuous vector field with an $\omega$-recurrent injective trajectory.

Besides the aim above, we produced a text on the basis of the qualitative theory of ordinary differential equations for continuous vector fields on manifolds. Such a theory can not be found in a single text like this one. In fact, just a few results are known for continuous vector fields an manifolds. Therefore, our contribution lies not only on the proof of a version of the Poincaré-Bendixson Theorem for $K^{2}$ but also on the development of the necessary theory for that. 



\section{Sumário}

Introdução

1 Campos vetoriais contínuos $\quad 1$

1.1 Conceitos e resultados básicos . . . . . . . . . . . . . . . . . . 1

1.2 Conjuntos limites . . . . . . . . . . . . . . . . . . . . 4

1.3 O Teorema de Poincaré-Bendixson no plano . . . . . . . . . . . . . 6

2 Campos vetoriais contínuos em variedades $\quad 15$

2.1 Teoria fundamental . . . . . . . . . . . . . . . . . . . . . 16

2.2 Conjuntos limites e recorrência _ . . . . . . . . . . . . . . . . 21

2.3 Campos vetoriais definidos por um recobrimento duplo orientado. . . . . . . 26

3 O Teorema de Poincaré-Bendixson na garrafa de Klein 31

3.1 Aspectos topológicos . . . . . . . . . . . . . . . . . . . . . . . . 31

3.2 O Teorema de Poincaré-Bendixson . . . . . . . . . . . . . . . . . . . . . . 39

4 Apêndice $\quad 51$

$\begin{array}{ll}\text { Referências Bibliográficas } & 53\end{array}$ 



\section{Introdução}

No estudo do comportamento assintótico dos sistemas dinâmicos, é essencial conhecermos a estrutura dos conjuntos para onde as trajetórias vão no futuro, ou de onde elas vieram no passado. A teoria de Poincaré-Bendixson trata do problema de se determinar a estrutura destes conjuntos, denominados conjuntos limites.

No final do século 19, H. Poincaré em [20] estabeleceu, sem dar uma prova completa, um teorema que descreve a estrutura dos conjuntos limites de trajetórias de campos vetoriais analíticos no plano. Em 1901, I. Bendixson provou, em [3], o teorema enunciado por H. Poincaré sob a hipótese mais fraca do campo vetorial ser apenas de classe $C^{1}$. A versão mais clássica do Teorema de Poincaré-Bendixson diz que se uma trajetória for limitada e o seu conjunto limite não contiver pontos singulares, então este será uma trajetória periódica.

Depois dos trabalhos pioneiros de H. Poincaré e de I. Bendixson, diversas generalizações do Teorema de Poincaré-Bendixson foram feitas. Por exemplo, o teorema foi generalizado para variedades bidimensionais em [10] e [23] (veja também [2]). Para fluxos contínuos, há o resultado de [11] e para semifluxos, o de [5]. No caso de semifluxos com impulsos, podemos citar [4]. Para campos vetoriais contínuos no plano, o Teorema de Poincaré-Bendixson encontra-se demonstrado em [12].

Em 1923, H. Kneser mostrou, em [13], que um fluxo contínuo na garrafa de Klein sem pontos singulares possui uma trajetória periódica. Na presença de pontos singulares, S. Kh. Aranson [1] e N. G. Markley [15] provaram este resultado de forma independente em 1969. Eles mostraram que fluxos contínuos na garrafa de Klein não possuem trajetória recorrente não-trivial. Em 1978, uma prova simplificada deste fato foi apresentada em [9] por C. Gutierrez.

O objetivo deste trabalho é apresentar uma versão do Teorema de Poincaré-Bendixson 
para campos vetoriais contínuos na garrafa de Klein. Além deste objetivo, procuramos produzir um texto que compreendesse a teoria qualitativa das equações diferenciais ordinárias para campos vetoriais contínuos em variedades, teoria esta que, até então, não podia ser encontrada num único texto.

Iniciamos este trabalho (Capítulo 1), relembrando alguns fatos básicos da teoria qualitativa das equações diferenciais ordinárias para campos vetoriais contínuos no $\mathbb{R}^{n}$. Em seguida, introduzimos o conceito de conjunto limite para trajetórias e algumas de suas propriedades. Ainda neste capítulo, enunciamos e provamos o Teorema de Poincaré-Bendixson para campos vetoriais contínuos no plano.

No Capítulo 2, definimos campos vetoriais contínuos em variedades e apresentamos, para este contexto, os resultados locais provenientes da teoria qualitativa da equações diferenciais ordinárias em $\mathbb{R}^{n}$ (Capítulo 1). Em seqüência, discutimos propriedades dos conjuntos limites e introduzimos um conceito mais fraco de recorrência para trajetórias. Na seção final deste capítulo, apresentamos a definição de levantamento de um campo vetorial contínuo por um recobrimento duplo orientado entre variedades compactas bidimensionais.

No Capítulo 3, tratamos dos aspectos topológicos da garrafa de Klein, os quais apresentamos em diversos resultados auxiliares. Finalmente, desenvolvemos e apresentamos uma versão do Teorema de Poincaré-Bendixson para campos vetoriais contínuos na garrafa de Klein $K^{2}$. Como conseqüência, obtemos o fato de que $K^{2}$ não possui campo vetorial contínuo com trajetória injetiva $\omega$-recorrente.

No Apêndice, incluímos um resultado que será útil nos Capítulos 2 e 3. 


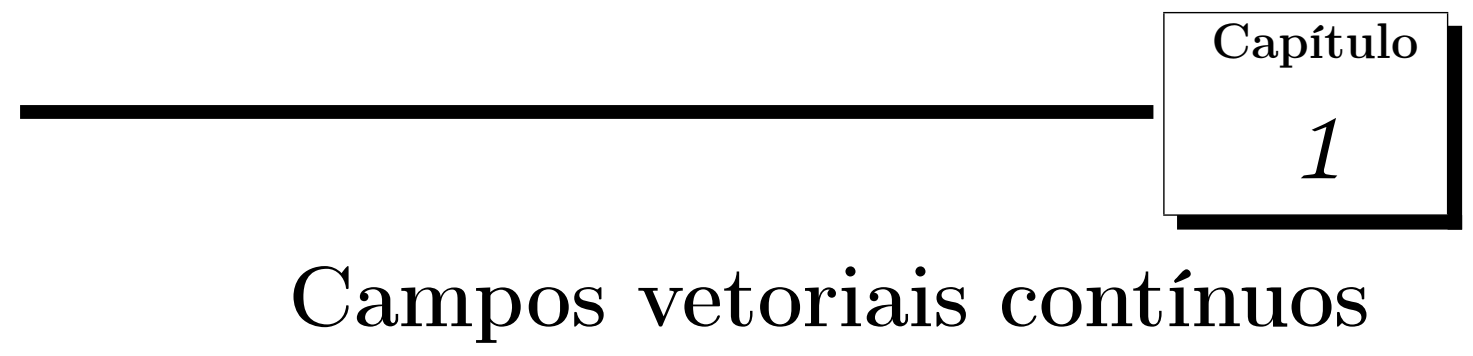

Iniciamos este capítulo resgatando algumas definições básicas e enunciando alguns resultados essenciais da teoria qualitativa das equações diferenciais ordinárias para campos vetoriais contínuos no $\mathbb{R}^{n}$, como por exemplo, os teoremas de existência e continuação de soluções. Em seguida, na segunda seção, apresentamos o conceito de conjunto limite para trajetórias e algumas de suas propriedades. Na última seção deste capítulo, demonstramos o Teorema de Poincaré-Bendixson para campos vetoriais contínuos no plano.

A principal referência para este capítulo é P. Hartman [12].

\subsection{Conceitos e resultados básicos}

Seja $U$ um subconjunto aberto do espaço euclidiano $\mathbb{R}^{n}$. Um campo vetorial contínuo em $U$ é uma aplicação $X: U \rightarrow \mathbb{R}^{n}$ contínua. Ao campo vetorial $X$ associamos a equação diferencial ordinária

$$
x^{\prime}=X(x), \quad\left(x^{\prime}=\frac{d x}{d t}\right) .
$$

As soluções desta equação, isto é, as aplicações diferenciáveis $\gamma: I \rightarrow U$ ( $I$ intervalo de $\mathbb{R}$ ) tais que

$$
\frac{d \gamma}{d t}(t)=X(\gamma(t))
$$

para todo $t \in I$, são chamadas curvas integrais de $X$ ou curvas integrais da equação diferencial (1.1).

Dados $p \in U$ e uma curva integral $\gamma: I \rightarrow U$ de $X$, diremos que $\gamma$ passa pelo ponto 
$p \in U$, se $0 \in I$ e $\gamma(0)=p$.

Observamos que se $\gamma: I \rightarrow U$ for uma curva integral de $X$ e $q=\gamma\left(t_{0}\right), t_{0} \in I$, então $\psi: J \rightarrow U$ definida por $\psi(t)=\gamma\left(t+t_{0}\right), t \in J$, também será uma curva integral de $X$, onde $J=\left\{r-t_{0}: r \in I\right\}$.

O teorema seguinte garante a existência de curvas integrais para um campo vetorial contínuo em $\mathbb{R}^{n}$.

Teorema 1.1.1 (Teorema de Peano) [12, Teorema 2.1, p. 10] Sejam $X: U \subset \mathbb{R}^{n} \rightarrow \mathbb{R}^{n}$ um campo vetorial contínuo e $p \in U$. Então existe pelo menos uma curva integral de $X$ passando por $p$.

Uma conseqüência importante do Teorema de Peano será apresentada no corolário seguinte.

Corolário 1.1.2 [12, Corolário 2.1, p. 11] Seja $X: U \subset \mathbb{R}^{n} \rightarrow \mathbb{R}^{n}$ um campo vetorial continuo limtado por uma constante $C>0$ e seja $K \subset U$ um conjunto compacto. Então existe $\alpha=\alpha(C, K)>0$ com a propriedade de que, para todo ponto $p \in K$, qualquer curva integral de $X$ passando por $p$ está definida no intervalo $[-\alpha, \alpha]$.

Uma curva integral $\gamma: I \rightarrow U$ de um campo vetorial contínuo $X: U \subset \mathbb{R}^{n} \rightarrow \mathbb{R}^{n}$ será dita máxima, se para toda curva integral $\psi: J \rightarrow U$ de $X \operatorname{com} I \subset J$ e $\gamma=\left.\psi\right|_{I}$, tivermos $I=J$. Em outras palavras, $\gamma$ será máxima, se não admitir nenhuma continuação (extensão) que também seja uma curva integral de $X$. Neste caso, o intervalo $I$ será chamado intervalo maximal de existência de $\gamma$.

O exemplo a seguir mostra que um campo vetorial contínuo pode admitir uma infinidade de curvas integrais máximas por um ponto.

Exemplo 1.1.3 Consideremos o campo vetorial contínuo $X: \mathbb{R} \rightarrow \mathbb{R}$ dado por $X(p)=3 p^{\frac{2}{3}}$, $p \in \mathbb{R}$. Para todo $c \geq 0$, a função $\gamma_{c}: \mathbb{R} \rightarrow \mathbb{R}$ definida por

$$
\gamma_{c}(t)= \begin{cases}(t-c)^{3}, & \text { se } t \geq c \\ 0, & \text { se } t \leq c\end{cases}
$$

é uma curva integral máxima de $X$ passando por 0 com intervalo maximal $\mathbb{R}$. 
O teorema seguinte diz que o intervalo maximal de existência de uma curva integral de um campo vetorial contínuo $X$ é aberto. Se tal intervalo for finito, a imagem da curva integral sairá de qualquer subconjunto compacto do domínio do campo $X$.

Teorema 1.1.4 [12, Teorema 3.1, p. 12] Sejam $X: U \subset \mathbb{R}^{n} \rightarrow \mathbb{R}^{n}$ um campo vetorial continuo e $\gamma$ uma curva integral de $X$ definida em algum intervalo. Então $\gamma$ pode ser continuada (como curva integral) até um intervalo aberto maximal. Além disso, se $\left(\omega_{-}, \omega_{+}\right)$ for um intervalo maximal de existência de $\gamma$ e $\omega_{+}<\infty$ (respectivamente $\left.\omega_{-}>-\infty\right)$, então $\gamma(t)$ tenderá à fronteira de $U$ quando $t \rightarrow \omega_{+}$(respectivamente $\left.t \rightarrow \omega_{-}\right)$, isto é, para todo compacto $K \subset U$, existirá $\epsilon=\epsilon(K)>0$ tal que se $t \in\left[\omega_{+}-\epsilon, \omega_{+}\right)$(respectivamente $\left.t \in\left(\omega_{-}, \omega_{-}+\epsilon\right]\right)$, então $\gamma(t) \notin K$.

Um resultado muito útil para campos vetoriais contínuos é dado no teorema seguinte e diz respeito ao limite de curvas integrais.

Teorema 1.1.5 [12, Teorema 3.2, p. 14] Seja $\left(X_{n}\right)_{n \in \mathbb{N}}$ uma seqüência de campos vetoriais continuos definidos no aberto $U \subset \mathbb{R}^{n}$ tal que $X_{n} \rightarrow X$, quando $n \rightarrow \infty$, uniformemente em subconjuntos compactos de $U$. Para cada $n \in \mathbb{N}$, seja $\gamma_{n}:\left(\omega_{n^{-}}, \omega_{n^{+}}\right) \rightarrow U$ uma curva integral máxima de $X_{n}$ passando por $p_{n} \in U$. Suponhamos que $p_{n} \rightarrow p \in U$ quando $n \rightarrow \infty$. Então existem uma curva integral máxima $\gamma:\left(\omega_{-}, \omega_{+}\right) \rightarrow U$ de X passando por $p$ e uma seqüência de inteiros positivos $\left(n_{k}\right)_{k \in \mathbb{N}}$ satisfazendo

$$
\gamma(t)=\lim _{k \rightarrow \infty} \gamma_{n_{k}}(t)
$$

onde o limite vale uniformemente em intervalos compactos de $\left(\omega_{-}, \omega_{+}\right)$.

A seguir, vamos introduzir mais definições e notações que serão utilizadas no decorrer deste capítulo.

Seja $X: U \subset \mathbb{R}^{n} \rightarrow \mathbb{R}^{n}$ um campo vetorial contínuo. Pelo Teorema de Peano e pelo Teorema de Continuação (Teorema 1.1.4), para cada ponto $p \in U$, existe uma curva integral $\gamma_{p}: I\left(\gamma_{p}\right) \rightarrow U$ de $X$ definida em algum intervalo maximal aberto $I\left(\gamma_{p}\right)$ que depende tanto da curva integral $\gamma_{p}$ como do ponto de partida $p$. Por simplicidade, identificamos a curva integral $\gamma_{p}$ com sua imagem $\left\{\gamma_{p}(t): t \in I\left(\gamma_{p}\right)\right\}$, a qual referimo-nos como a trajetória de $X$ passando por $p$ definida em $I\left(\gamma_{p}\right)$. 
A semitrajetória positiva (respectivamente, semitrajetória negativa) de $X$ passando por $p$ e contida em $\gamma_{p}$ é o conjunto

$$
\left.\gamma_{p}^{+}:=\left\{\gamma_{p}(t): t \in I\left(\gamma_{p}\right) \cap[0, \infty)\right\} \quad \text { (respectivamente, } \gamma_{p}^{-}:=\left\{\gamma_{p}(t): t \in I\left(\gamma_{p}\right) \cap(-\infty, 0]\right\}\right)
$$

Desta forma, $\gamma_{p}=\gamma_{p}^{-} \cup \gamma_{p}^{+}$.

Um arco de trajetória de $X$ é um subconjunto conexo de uma trajetória de $X$.

Diremos que $p \in U$ é um ponto singular ou uma singularidade (respectivamente um ponto regular) de $X$, se $X(p)=0$ (respectivamente $X(p) \neq 0$ ). Uma trajetória será dita regular, se ela não contiver pontos singulares. Uma trajetória $\gamma$ será periódica, se ela estiver definida em $\mathbb{R}$ e existir um $\tau>0$ tal que $\gamma(t+\tau)=\gamma(t)$ para todo $t \in \mathbb{R}$ e $\gamma\left(t_{1}\right) \neq \gamma\left(t_{2}\right)$, se $\left|t_{1}-t_{2}\right|<\tau$.

O conjunto aberto $U$, munido da decomposição em trajetórias de $X$, será dito retrato de fase de $X$. As trajetórias serão orientadas no sentido das curvas integrais de $X$; os pontos singulares serão munidos da orientação trivial.

Lembramos que trajetórias de campos vetoriais contínuos podem se auto-interceptar ou se interceptar mutuamente. Se uma trajetória se auto-interceptar, então ela naturalmente conterá uma trajetória periódica. Uma trajetória que não se auto-interceptar será dita injetiva.

\subsection{Conjuntos limites}

Sejam $U$ um subconjunto aberto de $\mathbb{R}^{n}$ e $X: U \rightarrow \mathbb{R}^{n}$ um campo vetorial contínuo.

Dada uma trajetória $\gamma$ de $X$ definida no intervalo maximal $I(\gamma)=\left(\omega_{-}(\gamma), \omega_{+}(\gamma)\right)$, se $\omega_{+}(\gamma)=\infty$, definiremos o conjunto $\omega$-limite de $\gamma$ como sendo o conjunto

$$
\omega(\gamma):=\left\{q \in U: \exists\left(t_{n}\right)_{n \in \mathbb{N}} \operatorname{com} t_{n} \rightarrow \infty \text { e } \gamma\left(t_{n}\right) \rightarrow q, \text { quando } n \rightarrow \infty\right\}
$$

Analogamente, se $\omega_{-}(\gamma)=\infty$, definiremos o conjunto $\alpha$-limite da trajetória $\gamma$ por

$$
\alpha(\gamma):=\left\{q \in U: \exists\left(t_{n}\right)_{n \in \mathbb{N}} \text { com } t_{n} \rightarrow-\infty \text { e } \gamma\left(t_{n}\right) \rightarrow q \text {, quando } n \rightarrow \infty\right\}
$$

O conjunto $\omega$-limite (respectivamente $\alpha$-limite) da trajetória $\gamma$ de $X$ também será chamado de conjunto $\omega$-limite da semitrajetória positiva $\gamma^{+} \subset \gamma$ (respectivamente conjunto $\alpha$-limite da semitrajetória negativa $\gamma^{-} \subset \gamma$ ) e será denotado por $\omega\left(\gamma^{+}\right)$(respectivamente 
$\left.\alpha\left(\gamma^{-}\right)\right)$

Os teoremas seguintes exibem algumas propriedades do conjunto $\omega$-limite de uma semitrajetória positiva. Resultados análogos valem para o conjunto $\alpha$-limite de uma semitrajetória negativa.

Teorema 1.2.1 [12, Teorema 1.1, p. 145] Sejam $X: U \subset \mathbb{R}^{n} \rightarrow \mathbb{R}^{n}$ um campo vetorial contínuo e $\gamma^{+}=\{\gamma(t): t \geq 0\}$ uma semitrajetória positiva de $X$. Então $\omega\left(\gamma^{+}\right)$é fechado. Se $\gamma^{+}$estiver contida num subconjunto compacto de $U$, então $\omega\left(\gamma^{+}\right)$será não vazio, compacto e conexo.

Na Figura 1.1, apresentamos um exemplo de uma semitrajetória $\gamma^{+}$cujo conjunto $\omega$ limite não é conexo por ser a união de duas retas paralelas. Isto ocorre, pois $\gamma^{+}$não está contida em nenhum subconjunto compacto de $\mathbb{R}^{2}$.

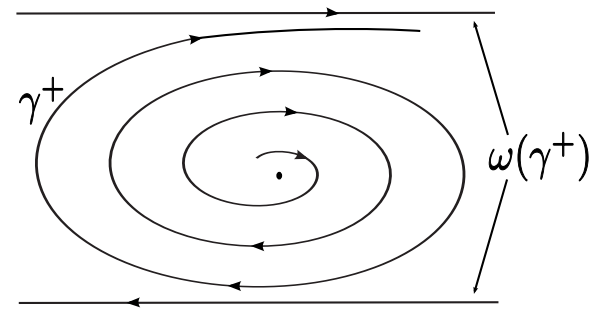

Figura 1.1: Um conjunto $\omega$-limite desconexo em $\mathbb{R}^{2}$.

A seguir, enunciaremos um resultado que garante um tipo de invariância para o conjunto $\omega$-limite, ou seja, ele garante que, para cada ponto no $\omega$-limite, existe pelo menos uma trajetória passando por esse ponto e inteiramente contida no $\omega$-limite.

Teorema 1.2.2 [12, Teorema 1.2, p. 145] Sejam $X: U \subset \mathbb{R}^{n} \rightarrow \mathbb{R}^{n}$ um campo vetorial contínuo e $\gamma^{+}=\{\gamma(t): t \geq 0\}$ uma semitrajetória positiva de $X$. Se $p \in \omega\left(\gamma^{+}\right)$, então $X$ terá pelo menos uma trajetória $\gamma_{p}$ passando por $p$ satisfazendo $\gamma_{p} \subset \omega\left(\gamma^{+}\right)$. Além disso, se $\gamma^{+}$estiver contida num subconjunto compacto de $U$, então $\gamma_{p}$ estará definida em $(-\infty, \infty)$ $e\left[\gamma_{p} \cup \alpha\left(\gamma_{p}\right) \cup \omega\left(\gamma_{p}\right)\right] \subset \omega\left(\gamma^{+}\right)$.

Corolário 1.2.3 Nas condições do Teorema 1.2.2, se $\omega\left(\gamma^{+}\right)$consistir de um único ponto $p \in U$, então $p$ será um ponto singular de $X$ e $\lim _{t \rightarrow \infty} \gamma^{+}(t)=p$. 


\subsection{O Teorema de Poincaré-Bendixson no plano}

O objetivo desta seção é enunciar e demonstrar o Teorema de Poincaré-Bendixson para campos vetoriais contínuos no plano. Este teorema, bem como os outros resultados desta seção, encontram-se no Capítulo VII de [12]. Por se tratarem de resultados pouco conhecidos para campos vetoriais contínuos, nós incluímos as demonstrações neste texto.

Antes de enunciarmos o Teorema de Poincaré-Bendixson, apresentaremos a definição de segmento transversal e provaremos algumas de suas propriedades.

No que segue, $U$ denotará um subconjunto aberto de $\mathbb{R}^{2}$ e $X$ denotará um campo vetorial contínuo em $U$.

Definição 1.3.1 Diremos que um segmento de reta fechado e limitado $\Sigma$ em $U \subset \mathbb{R}^{2}$ é um segmento transversal a $X$, se para todo ponto $p \in \Sigma, X(p)$ for um vetor não nulo e, junto com a direção de $\Sigma$, gerarem o plano $\mathbb{R}^{2}$.

Diremos que um segmento transversal $\Sigma$ passa por $p \in U$, se $p$ não for um ponto extremo de $\Sigma$.

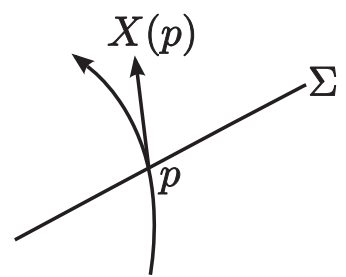

Figura 1.2: Segmento transversal $\Sigma$ ao campo $X$ por $p$.

Como conseqüência da definição anterior, todo ponto do segmento transversal $\Sigma$ é regular e $\Sigma$ não é tangente a qualquer trajetória que o intercepta.

Observamos que, devido ao fato de $\Sigma$ ser conexo e da continuidade do campo, as trajetórias de $X$ cruzam o segmento transversal $\Sigma$ sempre no mesmo sentido, digamos, da "esquerda" para a "direita", como mostra a Figura 1.3. 


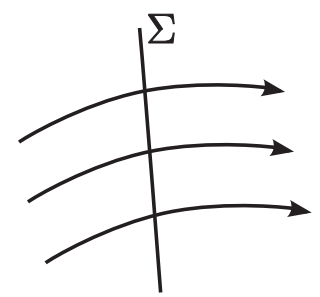

Figura 1.3: Trajetórias cruzando $\Sigma$.

O lema a seguir nos fornece propriedades de um segmento transversal a um campo vetorial contínuo $X$. Tal resultado foi extraído da demonstração do Teorema 4.1 de [12], p. 152, item $(a)$.

Lema 1.3.2 Nas condições acima sobre $U$ e $X$ temos:

(i) Se $p \in U$ for um ponto regular de $X$, então existirá um segmento transversal passando por $p$.

(ii) Seja $\Sigma$ um segmento transversal passando por um ponto $p \in U$. Então existem $\epsilon>0$ e uma vizinhança $V$ de $p$ em $\mathbb{R}^{2}$ tais que todas as trajetórias de $X$ passando por pontos de $V$ estão definidas em $[-\epsilon, \epsilon]$ e interceptam $\Sigma$ uma única vez para $|t| \leq \epsilon$.

(iii) Um arco de trajetória fechado e limitado de X intercepta um segmento transversal no máximo em um número finito de pontos.

\section{Demonstração:}

(i) Segue da definição de um segmento transversal e do fato de que $X$ é contínuo em $p$.

(ii) Seja $\delta>0$ arbitrário. Usando o Corolário 1.1.2, podemos escolher uma vizinhança $V$ de $p$ e um $\epsilon>0$ de forma que todas as trajetórias $\gamma_{q}$ de $X$ passando por $q \in V$ estejam definidas em $[-\epsilon, \epsilon]$ e de tal forma que $\gamma_{q}(t)$ diste de $q+t X(p)$ por no máximo $\delta|t|$ para $t \in[-\epsilon, \epsilon]$. Assim, se $V$ for suficientemente pequena, então $\gamma_{q}$ interceptará $\Sigma$ pelo menos uma vez, mas poderá interceptá-lo no máximo uma vez se $|t| \leq \epsilon$, pois as trajetórias de $X$ cruzam $\Sigma$ sempre no mesmo sentido. Isto demonstra $(i i)$.

(iii) Conseqüência imediata do item (ii).

Teorema 1.3.3 (Teorema de Poincaré-Bendixson) [12, Teorema 4.2, p. 154] Consideremos $X: U \subset \mathbb{R}^{2} \rightarrow \mathbb{R}^{2}$ um campo vetorial continuo e $\gamma^{+}=\{\gamma(t): t \geq 0\}$ uma semitraje- 
tória positiva injetiva de $X$ contida num compacto $K \subset U$. Suponhamos que $X$ possua um número finito de singularidades em $\omega\left(\gamma^{+}\right)$. Então ocorre uma das seguintes alternativas:

(i) $\omega\left(\gamma^{+}\right)$é uma trajetória periódica;

(ii) $\omega\left(\gamma^{+}\right)$é um ponto singular;

(iii) $\omega\left(\gamma^{+}\right)$é constituído de pontos singulares $\left\{p_{1}, p_{2}, \ldots, p_{n}\right\}$ e de uma seqüência (finita ou infinita) de arcos de trajetórias $\left\{\varphi(t):-\infty \leq \alpha_{-}<t<\alpha_{+} \leq \infty\right\}$ de X que não contêm pontos singulares, mas $\varphi\left(\alpha_{+}\right)=\lim _{t \rightarrow \alpha_{+}} \varphi(t)\left(\right.$ respectivamente $\left.\varphi\left(\alpha_{-}\right)=\lim _{t \rightarrow \alpha_{-}} \varphi(t)\right)$ existe e pertence ao conjunto $\left\{p_{1}, p_{2}, \ldots, p_{n}\right\}$.

A demonstração deste teorema será apresentada mais adiante.

Observação 1.3.4 Com a notação do Teorema 1.3.3, quando condições iniciais determinam soluções únicas de $X$, então $\alpha_{-}=-\infty$ e $\alpha_{+}=\infty$, pois $\varphi_{k}(t) \equiv p_{k}$ é a única solução de $X$ passando pelo ponto singular $p_{k}$.

O ingrediente essencial para a validade do Teorema de Poincaré-Bendixson é a cisão do domínio do campo em duas componentes por qualquer curva fechada simples, o que ocorre no plano e na esfera bidimensional mas não no toro bidimensional nem nos espaços euclidianos de dimensão maior do que 2. Este ingrediente é o famoso Teorema da Curva de Jordan. A sua prova pode ser encontrada em [17, p. 115].

Teorema 1.3.5 (Teorema da Curva de Jordan) Se $J \subset \mathbb{R}^{2}$ for uma curva fechada simples (isto é, J for a imagem homeomorfa de um círculo), então $\mathbb{R}^{2} \backslash J$ terá duas componentes conexas: $S_{i}\left(\right.$ limitada) e $S_{e}$ (não limitada), as quais terão J como fronteira comum.

Os resultados seguintes facilitarão a demonstração do Teorema de Poincaré-Bendixson. Em particular, os resultados contidos nos Lemas 1.3.6 e 1.3.7 podem ser encontrados na prova do Teorema 4.1 de [12], p. 152 - 153, itens (b) e (c) respectivamente.

Lema 1.3.6 Seja $\gamma^{+}=\{\gamma(t): t \geq 0\}$ uma semitrajetória positiva injetiva de um campo vetorial contínuo $X: U \subset \mathbb{R}^{2} \rightarrow \mathbb{R}^{2}$ e seja $\Sigma$ um segmento transversal a $X$. Se $\gamma^{+} \cap \Sigma \neq \varnothing$, então $\gamma^{+}$interceptará $\Sigma$ numa seqüência estritamente monótona. 


\section{Demonstração:}

Seja $D=\{t \geq 0: \gamma(t) \in \Sigma\}$. Decorre do Lema 1.3.2 que $D$ é discreto. Assim, podemos ordenar o conjunto

$$
D=\left\{t_{1}<t_{2}<\ldots<t_{n}<\ldots\right\}
$$

Como $D \neq \varnothing$, existe $p_{1}=\gamma\left(t_{1}\right)$. Definamos, caso exista, $p_{2}=\gamma\left(t_{2}\right)$. Por indução, definiremos $p_{n}=\gamma\left(t_{n}\right)$.

Sendo $\gamma^{+}$injetiva, então $p_{1} \neq p_{2}$. Para fixar as idéias, suponhamos que $p_{1}<p_{2}$. Se o ponto $p_{3}$ existir, vamos mostrar que $p_{3}>p_{2}$.

Consideremos a curva de Jordan $\Gamma$ formada pela união do segmento $\overline{p_{1} p_{2}} \subset \Sigma$ com o arco de trajetória $\left[p_{1}, p_{2}\right]_{\gamma}=\left\{\gamma(t): t_{1} \leq t \leq t_{2}\right\}$ de $X$, como mostra a Figura 1.4 abaixo.

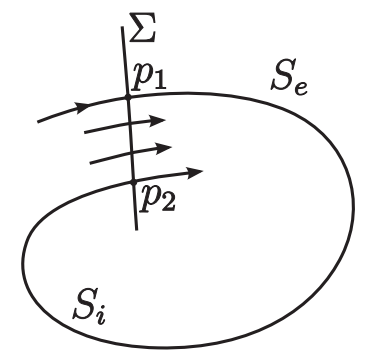

Figura 1.4: Curva de Jordan $\Gamma$.

Afirmamos que a semitrajetória positiva $\gamma_{p_{2}}^{+} \subset \gamma^{+}$fica contida na componente limitada $S_{i}$ de $\Gamma$. De fato, $\gamma_{p_{2}}^{+} \subset \gamma^{+}$não pode interceptar o arco $\left[p_{1}, p_{2}\right]_{\gamma}$, devido à injetividade de $\gamma^{+}$ (veja Figura 1.5(a)), e também não pode interceptar o segmento $\overline{p_{1} p_{2}} \subset \Sigma$, porque contraria o sentido no qual as trajetórias de $X$ cruzam $\Sigma$ (veja Figura $1.5(\mathrm{~b})$ ), o que demonstra a afirmação.

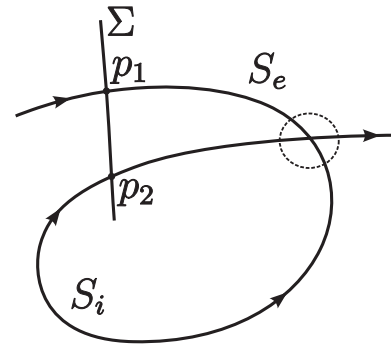

(a)

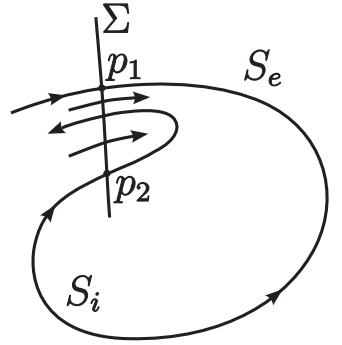

(b)

Figura 1.5: Configurações impossíveis de $\gamma_{p_{2}}^{+} \subset \gamma^{+}$. 
Pela nossa afirmação, caso $p_{3}$ exista, devemos ter $p_{1}<p_{2}<p_{3}$. Prosseguindo com este raciocínio, concluímos que a seqüência $\left(p_{n}\right)_{n \in \mathbb{N}}$ é estritamente monótona em $\Sigma$ e o lema está demonstrado.

Lema 1.3.7 Seja $X: U \subset \mathbb{R}^{2} \rightarrow \mathbb{R}^{2}$ um campo vetorial continuo e consideremos $\gamma^{+}=$ $\{\gamma(t): t \geq 0\}$ uma semitrajetória positiva injetiva de $X$ contida num compacto $K \subset U$. Então um segmento transversal $\Sigma$ intercepta $\omega\left(\gamma^{+}\right)$, no máximo, em um ponto.

\section{Demonstração:}

Suponhamos que exista um ponto regular $p \in \omega\left(\gamma^{+}\right) \cap \Sigma$. O item $(i i)$ do Lema 1.3 .2 e o Lema 1.3.6 implicam que a semitrajetória positiva $\gamma^{+}$intercepta $\Sigma$ segundo uma seqüência estritamente monótona. Assim, $\omega\left(\gamma^{+}\right) \cap \Sigma$ só poderá conter o ponto $p$, o que demonstra o lema.

Proposição 1.3.8 [12, Teorema 4.1, p. 151] Nas condições do Lema 1.3.7, se $\omega\left(\gamma^{+}\right)$contiver somente pontos regulares, então $\omega\left(\gamma^{+}\right)$será uma trajetória periódica.

\section{Demonstração:}

Seja $p \in \omega\left(\gamma^{+}\right)$. Pelo Teorema 1.2.2, existe uma trajetória $\gamma_{p}$ de $X$ passando por $p$, definida em $\mathbb{R}$ e satisfazendo $\gamma_{p} \subset \omega\left(\gamma^{+}\right)$. Além disso, $\omega\left(\gamma_{p}\right) \subset \omega\left(\gamma^{+}\right)$.

Sendo $\omega\left(\gamma^{+}\right)$compacto, então $\omega\left(\gamma_{p}\right) \neq \varnothing$. Seja $q \in \omega\left(\gamma_{p}\right)$. Assim, $q$ é um ponto regular, pois $\omega\left(\gamma^{+}\right)$não contém pontos singulares.

Consideremos um segmento transversal $\Sigma$ passando por $q$. Como $q \in \omega\left(\gamma_{p}\right) \subset \omega\left(\gamma^{+}\right)$, então a trajetória $\gamma_{p}$ intercepta $\Sigma$ em infinitos pontos e tais pontos, juntamente com $q$, pertencem a $\omega\left(\gamma^{+}\right)$. Pelo Lema 1.3.7, este pontos coincidem. Em particular, existem números reais positivos $t_{1}<t_{2}$ tais que $q=\gamma_{p}\left(t_{1}\right)=\gamma_{p}\left(t_{2}\right)$. Então $X$ possui uma trajetória periódica $\widetilde{\gamma}$ tal que $\widetilde{\gamma}(t)=\gamma_{p}(t)$ para $t \in\left[t_{1}, t_{2}\right]$. Como $\gamma_{p}$ não é constante em nenhum intervalo, podemos supor que $\widetilde{\gamma}\left(t_{0}\right) \neq \widetilde{\gamma}\left(t^{0}\right)$ para $0 \leq t_{0}<t^{0}<\tau$, onde $\tau=t_{2}-t_{1}>0$.

Afirmamos que $\omega\left(\gamma^{+}\right)=\widetilde{\gamma}$. De fato, suponhamos que $\omega\left(\gamma^{+}\right) \backslash \widetilde{\gamma} \neq \varnothing$. Então $\omega\left(\gamma^{+}\right) \backslash \widetilde{\gamma}$ tem um ponto de acumulação $\widetilde{p} \in \widetilde{\gamma}$, pois $\omega\left(\gamma^{+}\right)$é conexo. Seja $\Sigma_{\widetilde{p}}$ um segmento transversal passando por $\widetilde{p}$. Usando o Teorema 1.2 .2 e o fato de que toda vizinhança de $\widetilde{p}$ contém pontos de $\omega\left(\gamma^{+}\right) \backslash \widetilde{\gamma}$, então existe uma trajetória $\gamma_{p_{*}} \subset \omega\left(\gamma^{+}\right)$passando por um ponto $p_{*} \in \omega\left(\gamma^{+}\right) \backslash \widetilde{\gamma}$, definida em $\mathbb{R}$ e interceptando $\Sigma_{\widetilde{p}}$. O ponto de interseção é necessariamente $\widetilde{p}$, pelo Lema 1.3.7. 
Seja $\gamma_{p_{*}}(s) \in \widetilde{\gamma}$, quando $\gamma_{p_{*}}(t) \notin \widetilde{\gamma}$ para $t$ entre 0 e $s$. Como $\gamma_{p_{*}}(s)$ é um ponto regular, existe um segmento transversal $\Sigma_{*}$ passando por $\gamma_{p_{*}}(s)$. Então uma pequena translação de $\Sigma_{*}$, em uma direção conveniente, é um segmento transversal que intercepta $\widetilde{\gamma}$ e $\gamma_{p_{*}}$ em dois pontos distintos (veja Figura 1.6). Esta contradição com o Lema 1.3.7 demonstra nossa afirmação e, portanto, a proposição.

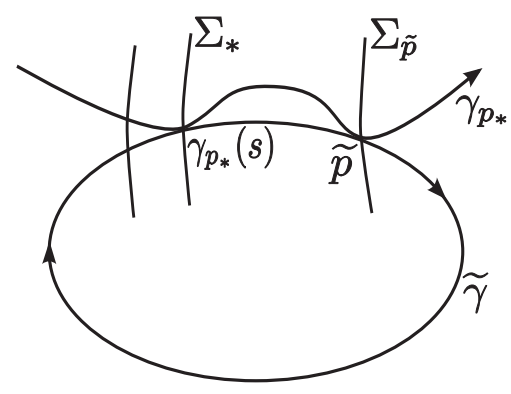

Figura 1.6: Os segmentos transversais $\Sigma_{\widetilde{p}}$ e $\Sigma_{*}$.

Como conseqüências imediatas da demonstração da proposição anterior, obtemos os seguintes resultados.

Corolário 1.3.9 Nas condições do Lema 1.3.7, se existir uma trajetória periódica $\widetilde{\gamma}$ em $\omega\left(\gamma^{+}\right)$consistindo somente de pontos regulares, então $\omega\left(\gamma^{+}\right)=\widetilde{\gamma}$.

Corolário 1.3.10 Seja $\gamma^{+}$uma semitrajetória positiva injetiva de um campo vetorial contínuo $X: U \subset \mathbb{R}^{2} \rightarrow \mathbb{R}^{2}$ e seja $p \in \omega\left(\gamma^{+}\right)$um ponto regular. Então existe uma vizinhança $V$ de $p$ em $U$ tal que $X$ possui uma única trajetória passando por $p$ em $\omega\left(\gamma^{+}\right) \cap V$.

Agora, estamos em condições de apresentar uma prova do Teorema de Poincaré-Bendixson (Teorema 1.3.3). Veja [12, Teorema 4.2, p. 154].

\section{Demonstração do Teorema de Poincaré-Bendixson (Teorema 1.3.3):}

Se $\omega\left(\gamma^{+}\right)$contiver somente pontos regulares, então, pela Proposição 1.3.8, $\omega\left(\gamma^{+}\right)$será uma trajetória periódica.

Se $\omega\left(\gamma^{+}\right)$não contiver pontos regulares, então $\omega\left(\gamma^{+}\right)$será um ponto singular, pois $X$ tem um número finito de pontos singulares em $\omega\left(\gamma^{+}\right)$e $\omega\left(\gamma^{+}\right)$é conexo.

Suponhamos, então, que $\omega\left(\gamma^{+}\right)$contenha pontos regulares e singulares. Pelo Teorema 1.2.2, para cada ponto regular $p$ em $\omega\left(\gamma^{+}\right)$, existe uma trajetória $\gamma_{p}$ de $X$ passando por $p$, definida em $\mathbb{R}$ e satisfazendo $\gamma_{p} \subset \omega\left(\gamma^{+}\right)$. 
Afirmamos que $\gamma_{p}$ contém um arco de trajetória especificado em (iii). De fato, para demonstrar isto, iremos considerar somente a semitrajetória positiva $\gamma_{p}^{+} \subset \gamma_{p}$, pois o tratamento para a semitrajetória negativa $\gamma_{p}^{-} \subset \gamma_{p}$ é análogo. Então uma das seguintes alternativas ocorre:

(a) existe um primeiro número real positivo $t=\alpha_{+}$tal que $\gamma_{p}\left(\alpha_{+}\right)$é um ponto singular (logo pertencente a $\left.\left\{p_{1}, \ldots, p_{n}\right\}\right)$ ou

(b) $\gamma_{p}^{+} \subset \gamma_{p}$ não contém pontos singulares.

No caso $(b)$, mostraremos que $\omega\left(\gamma_{p}^{+}\right)$é um ponto singular. Suponhamos que exista um ponto regular em $\omega\left(\gamma_{p}^{+}\right)$. Procedendo como na demonstração da Proposição 1.3.8, então $\gamma_{p}^{+} \subset$ $\gamma_{p}$ contém uma trajetória periódica $\widetilde{\gamma}$. Como $\widetilde{\gamma} \subset \omega\left(\gamma^{+}\right)$não contém pontos singulares, o Corolário 1.3.9 implica que $\omega\left(\gamma^{+}\right)=\widetilde{\gamma}$. Mas isto contradiz a hipótese sobre $\omega\left(\gamma^{+}\right)$. Portanto, $\omega\left(\gamma_{p}^{+}\right)$só contém pontos singulares e, como ele é conexo, então $\omega\left(\gamma_{p}^{+}\right)$é um ponto singular $p_{k}$ pertencente a $\left\{p_{1}, \ldots, p_{n}\right\}$. Pelo Corolário 1.2.3, $\lim _{t \rightarrow \infty} \gamma_{p}(t)=p_{k}$ e isto demonstra nossa afirmação.

Resta-nos mostrar que o conjunto dos arcos de trajetórias contidos em $\omega\left(\gamma^{+}\right)$é enumerável. Usando o Corolário 1.3.10, então, para cada ponto regular $p \in \omega\left(\gamma^{+}\right)$, o arco de trajetória contido em $\omega\left(\gamma^{+}\right)$passando por $p$ é único.

Podemos supor que $\gamma^{+}$não contenha nenhum dos pontos singulares $\left\{p_{1}, \ldots, p_{n}\right\}$, pela injetividade de $\gamma^{+}$. Além disso, se $p \in \omega\left(\gamma^{+}\right)$for um ponto regular, então $\gamma^{+}(t) \neq p$ para todo $t \geq 0$, pois a seqüência de intercecções de $\gamma^{+}$com um segmento transversal passando por $p$ tende a $p$ monotonicamente. Assim, $\gamma^{+}$e $\omega\left(\gamma^{+}\right)$não têm pontos em comum.

Suponhamos, por contradição, que o conjunto dos arcos de trajetórias contidos em $\omega\left(\gamma^{+}\right)$ não seja enumerável. Como $X$ tem um número finito de pontos singulares em $\omega\left(\gamma^{+}\right)$, existe um subconjunto não enumerável destes arcos que unem dois pontos singulares $p_{1}$ e $p_{2}$ (distintos ou não). A união de quaisquer um ou dois destes arcos forma uma curva de Jordan. Como existe no máximo um conjunto enumerável de curvas de Jordan com pares de interiores disjuntos, então existem três tais curvas disjuntas, digamos $J_{1}, J_{2}$ e $J_{3}$, tais que $J_{3}$ está contida no fecho do interior de $J_{1}$ e também no fecho do interior de $J_{2}$ (veja Figura 1.7). Isto é impossível, pois como $\gamma^{+}$não intercepta as curvas $J_{1}$ e $J_{2}$, então $\gamma^{+}$está ou entre $J_{1}$ e $J_{2}$ ou entre $J_{2}$ e $J_{3}$ e o teorema está demonstrado. 


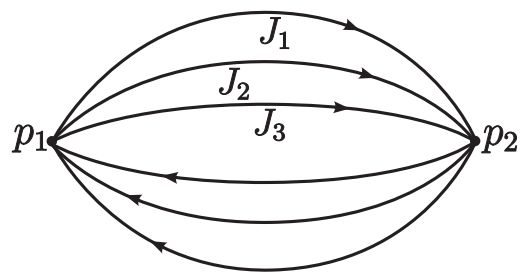

Figura 1.7: Curvas de Jordan $J_{1}, J_{2}$ e $J_{3}$.

No próximo teorema, iremos mostrar que o conjunto $\omega$-limite de uma semitrajetória positiva (não necessariamente injetiva) contém uma trajetória periódica ou um ponto singular.

Teorema 1.3.11 [12, Teorema 4.3, p. 155] Sejam $X: U \subset \mathbb{R}^{2} \rightarrow \mathbb{R}^{2}$ um campo vetorial continuo e $\gamma^{+}=\{\gamma(t): t \geq 0\}$ uma semitrajetória positiva de $X$ contida num compacto $K \subset U$. Então $\omega\left(\gamma^{+}\right)$contém uma trajetória periódica ou um ponto singular.

\section{Demonstração:}

Suponhamos que $\omega\left(\gamma^{+}\right)$não contenha pontos singulares. Seja $p \in \omega\left(\gamma^{+}\right)$. Pelo Teorema 1.2.2, existe uma trajetória $\gamma_{p}$ de $X$ passando por $p$, definida em $\mathbb{R}$ e satisfazendo $\gamma_{p} \subset \omega\left(\gamma^{+}\right)$. Além disso, $\omega\left(\gamma_{p}\right) \subset \omega\left(\gamma^{+}\right)$. Se $\gamma_{p}$ for uma trajetória injetiva, então $\omega\left(\gamma_{p}\right)$ será uma trajetória periódica $\widetilde{\gamma}$, pela Proposição 1.3.8. Se $\gamma_{p}\left(t_{1}\right)=\gamma_{p}\left(t_{2}\right)$ para números reais positivos $t_{1}<t_{2}$, então $\gamma_{p}$ conterá uma trajetória periódica $\widetilde{\gamma}$ tal que $\widetilde{\gamma}(t)=\gamma_{p}(t)$ para $t \in\left[t_{1}, t_{2}\right]$. Em ambos os casos, $\omega\left(\gamma^{+}\right)$conterá uma trajetória periódica $\widetilde{\gamma}$. Isto demonstra o teorema.

A demonstração do próximo resultado utiliza o conceito de índice de um ponto singular no plano e pode ser encontrada em [12], Teorema 3.1, p. 150.

Teorema 1.3.12 Sejam $X: U \subset \mathbb{R}^{2} \rightarrow \mathbb{R}^{2}$ um campo vetorial contínuo e $\gamma$ uma trajetória periódica de $X$ que não contém pontos singulares. Se o interior de $\gamma$ estiver contido em $U$, então existirá um ponto singular de $X$ no interior de $\gamma$. 



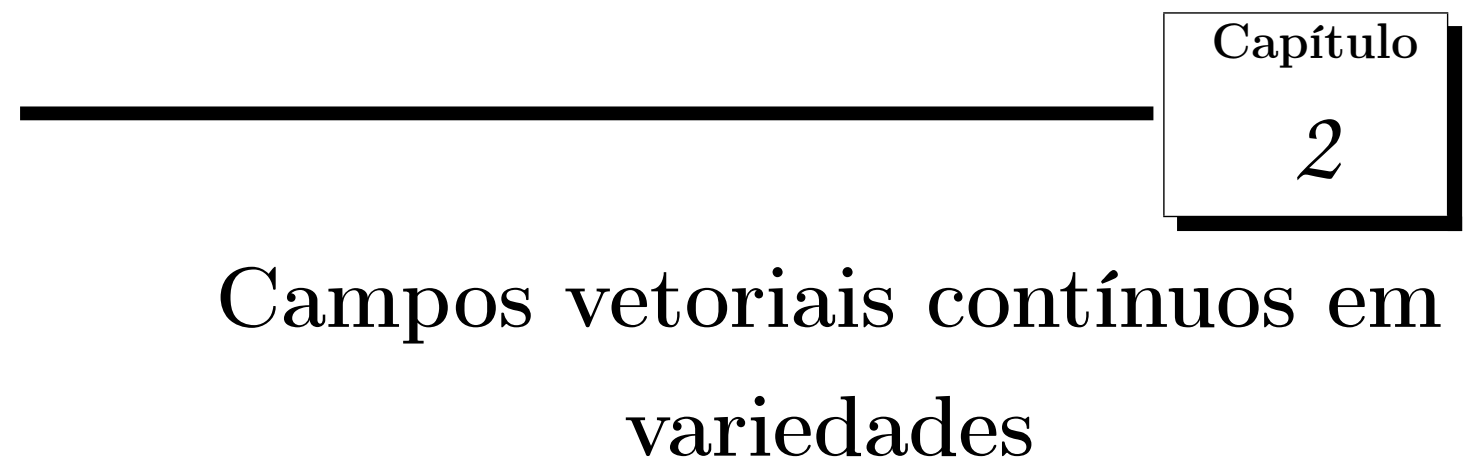

Para a teoria qualitativa das equações diferenciais ordinárias para campos vetoriais de classe $C^{1}$ em variedades, indicamos o livro [2]. No caso de campos vetoriais contínuos, não temos o conhecimento de um livro-texto que aborde esta teoria. Por este motivo, resolvemos desenvolver e apresentar esta teoria neste capítulo.

Na primeira seção deste capítulo, definimos campos vetoriais contínuos em variedades e apresentamos, para este contexto, os resultados locais provenientes da teoria qualitativa das equações diferenciais ordinárias em $\mathbb{R}^{n}$.

Em seguida, na segunda seção deste capítulo, discutimos propriedades dos conjuntos limites. No caso de fluxos contínuos, a invariância dos conjuntos $\alpha$ e $\omega$-limites segue da dependência contínua das trajetórias com relação às condições iniciais. No caso de campos vetoriais contínuos, não podemos contar com a dependência contínua das trajetórias com relação às condições iniciais. Entretanto, é possível garantir um tipo de invariância para os conjuntos $\alpha$ e $\omega$-limites. Provamos que, para todo ponto no $\omega$-limite, existe pelo menos uma trajetória acompanhada passando por esse ponto e inteiramente contida nele (veja Definição 2.2.2 e Proposição 2.2.3 a seguir). Também, pelo fato de não podermos contar com a unicidade das trajetórias de campos vetoriais contínuos, foi preciso introduzir um conceito mais fraco de recorrência para trajetórias.

Na última seção deste capítulo, recordamos a definição de recobrimento duplo orientado entre variedades compactas bidimensionais e a definição de levantamento de um campo ve- 
torial contínuo por este recobrimento. Mostramos que as trajetórias de um dado campo vetorial contínuo estão relacionadas com as trajetórias do seu levantamento. Também apresentamos uma relação entre os conjuntos limites dessas trajetórias (Lema 2.3.7).

\subsection{Teoria fundamental}

Seja $M$ uma variedade de dimensão $n$, sem bordo e classe $C^{\infty}$. Para cada $p \in M$, seja $T_{p} M$ o espaço de vetores tangentes a $M$ em $p$ e seja

$$
T M=\left\{\left(p, v_{p}\right): p \in M, v_{p} \in T_{p} M\right\},
$$

o espaço fibrado tangente de $M$.

Denotemos por $\pi_{1}: T M \rightarrow M$ a projeção dada por $\pi_{1}\left(p, v_{p}\right)=p$.

Definição 2.1.1 Um campo vetorial contínuo em $M$ é uma aplicação $X: M \rightarrow T M$ contínua tal que $\pi_{1} \circ X: M \rightarrow M$ é a identidade, ou seja, $X$ é uma aplicação contínua que a cada ponto $p \in M$ associa um vetor $X(p) \in T_{p} M$.

No que segue, $\mathfrak{X}(M)$ denotará o espaço dos campos vetoriais contínuos em $M$.

Definição 2.1.2 Uma curva integral de $X \in \mathfrak{X}(M)$ passando por um ponto $p \in M$ é uma aplicação $\gamma: I \rightarrow M$ de classe $C^{1}$ tal que $\gamma(0)=p$ e $\gamma^{\prime}(t)=X(\gamma(t))$ para todo $t \in I$, onde I é um intervalo de $\mathbb{R}$ contendo 0 .

Numa carta local $x: U \subset M \rightarrow \mathbb{R}^{n}$ de $M$, um campo vetorial $X \in \mathfrak{X}(M)$ é representado pelo campo vetorial contínuo $x_{*}(X): x(U) \subset \mathbb{R}^{n} \rightarrow \mathbb{R}^{n}$ dado por

$$
x_{*}(X)(p)=d x_{x^{-1}(p)} \cdot X\left(x^{-1}(p)\right), p \in x(U),
$$

onde $d x$ e $x^{-1}$ denotam, respectivamente, as aplicações derivada e inversa da carta local $x$. Diremos que $x_{*}(X)$ é a expressão de $X$ na carta local $x: U \subset M \rightarrow \mathbb{R}^{n}$.

Um fato simples de verificar é o seguinte. Seja $\gamma: I \rightarrow U \subset M$ uma aplicação de classe $C^{1}$. Então $\gamma$ será uma curva integral de $X$ se, e somente se, $x \circ \gamma: I \rightarrow x(U) \subset \mathbb{R}^{n}$ for uma curva integral de $x_{*}(X)$. Conjugando o fato acima com o teorema de existência de curvas integrais de uma equação diferencial ordinária em $\mathbb{R}^{n}$ (Teorema de Peano), podemos enunciar o resultado seguinte. 
Teorema 2.1.3 Sejam $X \in \mathfrak{X}(M)$ e $p \in M$. Então existe pelo menos uma curva integral de $X$ passando por $p$.

Lema 2.1.4 Seja $X \in \mathfrak{X}(M)$. Para cada ponto $p \in M$, existem uma vizinhança $V \subset M$ de $p$ e um número real $\alpha>0$ tais que todas as curvas integrais de $X$ passando por um dado ponto de $V$ estão definidas no intervalo $[-\alpha, \alpha]$.

\section{Demonstração:}

Basta aplicarmos o Corolário 1.1.2 em uma expressão de $X$, cuja carta local associada contém o ponto $p$ no seu domínio.

O lema seguinte diz que uma curva integral pode ser reparametrizada para mudar seu ponto inicial.

Lema 2.1.5 Sejam $X \in \mathfrak{X}(M)$ e $\gamma: I \rightarrow M$ uma curva integral de $X$ passando por um ponto $p \in M$. Fixemos $t_{0} \in I$ e consideremos $\tilde{I}=\left\{t \in \mathbb{R}: t+t_{0} \in I\right\}$. Então a curva $\widetilde{\gamma}: \tilde{I} \rightarrow M$ definida por $\widetilde{\gamma}(t)=\gamma\left(t+t_{0}\right)$ é uma curva integral de $X$ passando por $\gamma\left(t_{0}\right)$.

\section{Demonstração:}

Aplicação direta da Regra da Cadeia em cartas locais.

Definição 2.1.6 Uma curva integral $\gamma: I \rightarrow M$ de $X \in \mathfrak{X}(M)$ será dita máxima, se para toda curva integral $\psi: J \rightarrow M$ de $X$ tal que $I \subset J$ e $\gamma=\left.\psi\right|_{I}$ tivermos $I=J$ e conseqüentemente $\gamma=\psi$. Neste caso, I será chamado intervalo maximal de existência de $\gamma$.

Vamos prosseguir com nossa exposição a fim de apresentarmos um teorema que diz que toda curva integral de um campo vetorial contínuo em uma variedade compacta está definida em $\mathbb{R}$. Antes, porém, demonstraremos o lema seguinte.

Lema 2.1.7 Sejam $X \in \mathfrak{X}(M)$ e $\gamma$ uma curva integral máxima de $X$. Se o intervalo de existência de $\gamma$ não for todo o $\mathbb{R}$, então sua imagem não estará contida em nenhum subconjunto compacto de $M$.

\section{Demonstração:}

Para fixar as idéias, suponhamos que o intervalo maximal de existência de $\gamma$ seja $(a, b)$ com $b<\infty$. O argumento para o caso $a>-\infty$ é análogo. 
Suponhamos, por contradição, que $\gamma(a, b)$ esteja contido num compacto $K \subset M$. Consideremos uma seqüência $\left(t_{n}\right)_{n \in N}$ em $(a, b)$, com $t_{n} \rightarrow b$ quando $n \rightarrow \infty$. Como $\gamma(a, b) \subset K$ e $K$ é compacto, podemos supor, passando a uma subseqüência, que $\gamma\left(t_{n}\right)$ converge para $p \in K$.

Sejam $V \subset M$ uma vizinhança de $p$ e $\alpha>0$ dados pelo Lema 2.1.4. Tomemos $n_{0} \in \mathbb{N}$ tal que $b-t_{n_{0}}<\frac{\alpha}{2}$ e $\gamma\left(t_{n_{0}}\right) \in V$. O Lema 2.1.4 implica que todas as curvas integrais de $X$ passando por $\gamma\left(t_{n_{0}}\right)$ estão definidas em $(-\alpha, \alpha)$. Em particular, a curva integral $\gamma$ pode ser estendida ao intervalo $\left(a, t_{n_{0}}+\alpha\right) \supset(a, b)$. Mas isto contradiz a maximalidade de $\gamma$ e o lema está demonstrado.

Teorema 2.1.8 Suponhamos que $M$ seja uma variedade compacta. Então as curvas integrais de $X \in \mathfrak{X}(M)$ estão definidas em $\mathbb{R}$.

\section{Demonstração:}

Sendo $M$ compacta, o Lema 2.1.7 implica que nenhuma curva integral pode ter intervalo maximal de existência que não seja todo o $\mathbb{R}$, pois a imagem de qualquer curva integral está contida no conjunto compacto $M$.

O teorema seguinte será utilizado na próxima seção para provar um tipo de invariância para os conjuntos limites das trajetórias sobre campos vetoriais contínuos em variedades.

Teorema 2.1.9 Seja $\left(X_{n}\right)_{n \in \mathbb{N}} \in \mathfrak{X}(M)$ uma seqüência de campos vetoriais contínuos definidos em uma variedade compacta $M$ tal que $X_{n} \rightarrow X$, quando $n \rightarrow \infty$, uniformemente em M. Para cada $n \in \mathbb{N}$, seja $\gamma_{n}: \mathbb{R} \rightarrow M$ uma curva integral de $X_{n}$ passando por $p_{n} \in M$. Suponhamos que $p_{n} \rightarrow p \in M$, quando $n \rightarrow \infty$. Então existem uma curva integral $\gamma: \mathbb{R} \rightarrow M$ de $X$ passando por p e uma seqüência de inteiros positivos $\left(n_{k}\right)_{k \in \mathbb{N}}$ satisfazendo

$$
\gamma(t)=\lim _{k \rightarrow \infty} \gamma_{n_{k}}(t),
$$

onde o limite vale uniformemente em intervalos compactos de $\mathbb{R}$.

\section{Demonstração:}

Mostremos, inicialmente, que 
(a) se $x: U \subset M \rightarrow \mathbb{R}^{n}$ for uma carta local de $M$, então, para cada conjunto compacto $V \subset U$, existirá $\alpha>0$ tal que todas as curvas integrais de $X$ e $X_{n}, n \in \mathbb{N}$, passando por um dado ponto de $V$ estão definidas no intervalo $[-\alpha, \alpha]$.

Com efeito, sejam $x_{*}(X)$ e $x_{*}\left(X_{n}\right)$ as expressões de $X$ e $X_{n}, n \in \mathbb{N}$, na carta local $x: U \subset$ $M \rightarrow \mathbb{R}^{n}$ respectivamente.

Sendo $x(V) \subset x(U)$ compacto, existe um conjunto aberto $W$ tal que $x(V) \subset W \subset$ $\bar{W} \subset x(U)$. Como $X_{n} \rightarrow X$, quando $n \rightarrow \infty$, uniformemente em $M$ e $d x$ é uniformemente contínua no conjunto compacto $X\left(x^{-1}(\bar{W})\right)$, então $x_{*}\left(X_{n}\right) \rightarrow x_{*}(X)$, quando $n \rightarrow \infty$, uniformemente em $\bar{W}$. Daí, existe $C>0$ tal que $\left|x_{*}(X)\right|<C$ e $\left|x_{*}\left(X_{n}\right)\right|<C, n \in \mathbb{N}$, em $\bar{W}$, onde $|\cdot|$ é a norma euclidiana em $\mathbb{R}^{n}$. Pelo Corolário 1.1.2, existe $\alpha>0$, independente de $n$, tal que todas as curvas integrais de $x_{*}(X)$ e $x_{*}\left(X_{n}\right)$ passando por um dado ponto de $x(V)$ estão definidas no intervalo $[-\alpha, \alpha]$. Isto demonstra $(a)$.

Seja $\left(U_{n}\right)_{n \in \mathbb{N}}$ uma cobertura aberta de $M$ constituída por domínios de cartas locais $x_{n}: U_{n} \subset M \rightarrow \mathbb{R}^{n}$. Tomemos uma cobertura aberta $\left(V_{n}\right)_{n \in \mathbb{N}}$ de $M$ tal que $\overline{V_{n}} \subset U_{n}$, para todo $n \in \mathbb{N}$. Como $M$ é compacta, $\left(V_{n}\right)_{n \in \mathbb{N}}$ possui uma subcobertura finita, digamos $M=\cup_{i=1}^{l} V_{i}$.

Para fixar as idéias, suponhamos que $p \in V_{1}$. Como $p_{n} \rightarrow p$, quando $n \rightarrow \infty$, podemos assumir que $p_{n} \in V_{1}$, para todo $n \in \mathbb{N}$.

Iremos construir $\gamma$ definida no intervalo $[0, \infty)$. A construção no intervalo $(-\infty, 0]$ é análoga.

Por $(a)$, para cada $i \in\{1, \ldots, l\}$, existe $\alpha_{i}>0$ tal que todas as curvas integrais de $X$ e $X_{n}, n \in \mathbb{N}$, passando por um dado ponto de $V_{i}$ estão definidas no intervalo $\left[-\alpha_{i}, \alpha_{i}\right]$. Seja $\alpha=\min \left\{\alpha_{i}: i=1, \ldots, l\right\}$.

Aplicando o Teorema 1.1.5 aos campos $x_{1_{*}}(X)$ e $x_{1_{*}}\left(X_{n}\right), n \in \mathbb{N}$, obtemos uma seqüência de inteiros positivos $\left(n_{k}\right)_{k \in \mathbb{N}}$ tal que o limite (2.1) vale uniformemente em [0, $\left.\alpha\right]$ e é uma curva integral de $X$ passando por $p$. Se o ponto $\gamma(\alpha)$ pertencer a $V_{1}$, então a seqüência $\left(n_{k}\right)_{k \in \mathbb{N}}$ poderá ser substituída por uma subseqüência, que ainda denominaremos por $\left(n_{k}\right)_{k \in \mathbb{N}}$, tal que o limite (2.1) vale uniformemente em $[\alpha, 2 \alpha]$ e é uma curva integral de $X$.

Podemos supor que este processo pode ser repetido $j$ vezes, onde $\gamma(m \alpha) \in V_{1}$ para $m=0, \ldots, j-1$ e $\gamma(j \alpha) \notin V_{1}$, pois, caso contrário, pelo Teorema 1.1.5, não haveria o que demonstrar. Neste caso, sejam $t_{1}=j \alpha$ e $r \in\{2, \ldots, l\}$ tais que $\gamma\left(t_{1}\right) \in V_{r}$. Repetindo o 
argumento acima, obtemos $\gamma$ definida no intervalo $\left[t_{1}, t_{1}+j_{1} \alpha\right]$, onde $\gamma\left(t_{1}+m \alpha\right) \in V_{r}$ para $m=0, \ldots, j_{1}-1$ e $\gamma\left(j_{1} \alpha\right) \notin V_{r}$.

Consideremos $t_{2}=t_{1}+j_{1} \alpha$. Prosseguindo desta maneira, obtemos uma seqüência estritamente crescente de números reais $\left(t_{n}\right)_{n \in \mathbb{N}}$ e uma seqüência de subseqüências sucessivas de inteiros

$$
\begin{aligned}
& n_{1_{1}}<n_{1_{2}}<\ldots \\
& n_{2_{1}}<n_{2_{2}}<\ldots \\
& \ldots \ldots \ldots \ldots
\end{aligned}
$$

tais que o limite $(2.1)$ vale uniformemente em $\left[0, t_{m}\right]$, se $n_{k}=n_{m_{k}}$. Isto mostra que $\gamma$ está definida em $[0, \infty)$ e o método da diagonal de Cantor nos fornece uma seqüência $\left(n_{k}\right)_{k \in \mathbb{N}}$ satisfazendo (2.1). O teorema está demonstrado.

De agora em diante, vamos nos restringir aos campos vetoriais contínuos sobre variedades compactas bidimensionais. Por variedade compacta bidimensional entende-se uma variedade bidimensional a qual é compacta, conexa e de classe $C^{\infty}$. Salvo menção em contrário, assumiremos que qualquer variedade compacta bidimensional é sem bordo.

Agora, introduziremos algumas definições e notações que serão utilizadas até o final deste trabalho.

Sejam $M$ uma variedade compacta bidimensional e $X \in \mathfrak{X}(M)$. Pelo Teorema 2.1.8, para cada ponto $p \in M$, existe pelo menos uma curva integral $\gamma_{p}: \mathbb{R} \rightarrow M$ de $X$. Por simplicidade, identificamos a curva $\gamma_{p}$ com sua imagem $\left\{\gamma_{p}(t): t \in \mathbb{R}\right\}$, a qual referimonos como uma trajetória de $X$ passando por $p$. A semitrajetória positiva (respectivamente, semitrajetória negativa) de $X$ passando por $p$ e contida em $\gamma_{p}$ é o conjunto

$$
\left.\gamma_{p}^{+}:=\left\{\gamma_{p}(t): t \geq 0\right\} \quad \text { (respectivamente, } \gamma_{p}^{-}:=\left\{\gamma_{p}(t): t \leq 0\right\}\right)
$$

Desta forma, $\gamma_{p}=\gamma_{p}^{-} \cup \gamma_{p}^{+}$.

Um arco de trajetória de $X$ é um subconjunto conexo de uma trajetória de $X$.

Diremos que $p \in M$ é um ponto singular ou uma singularidade (respectivamente um ponto regular) de $X$, se $X(p)=0$ (respectivamente $X(p) \neq 0$ ). Uma trajetória será dita regular, se ela não contiver pontos singulares. Uma trajetória $\gamma$ será periódica, se existir $\tau>0$ tal que $\gamma(t+\tau)=\gamma(t)$ para todo $t \in \mathbb{R}$. 
Recordamos que trajetórias de campos vetoriais contínuos podem se auto-interceptar ou se interceptar mutuamente. Se uma trajetória se auto-interceptar, então ela naturalmente conterá uma trajetória periódica. Uma trajetória que não se auto-interceptar será dita injetiva.

Uma subvariedade unidimensional $\Sigma$ de $M$ será dita uma transversal ao campo vetorial $X$, se ela não contiver nenhuma singularidade de $X$ e for transversal a cada trajetória de $X$. Uma transversal será dita um segmento transversal, se for homeomorfa a um subintervalo fechado não-degenerado de $\mathbb{R}$. Assim, um segmento transversal tem uma ordenação total $" \leq "$ induzida pela ordenação total do intervalo. Diremos que um segmento transversal $\Sigma$ passa por $p \in M$, se $p$ não for um ponto extremo de $\Sigma$. Desta maneira, se $\Sigma$ for um segmento transversal passando por $p$, então $\Sigma \backslash\{p\}$ terá duas componentes conexas.

Neste trabalho, vamos supor que todas as trajetórias de campos vetoriais contínuos em variedades compactas bidimensionais sejam regulares, a menos das trajetórias dadas por pontos singulares. De fato, isto pode ser feito, sem perda de generalidade, pois podemos considerar um outro campo vetorial contínuo com o mesmo conjunto de singularidades e o mesmo retrato de fase, mas tendo somente trajetórias regulares, exceto para as trajetórias que são pontos singulares (veja o Apêndice).

\subsection{Conjuntos limites e recorrência}

Sejam $M$ uma variedade compacta bidimensional e $X \in \mathfrak{X}(M)$. Definimos o conjunto $\omega$-limite de uma trajetória $\gamma$ de $X$ como sendo o conjunto

$$
\omega(\gamma):=\left\{q \in M: \exists\left(t_{n}\right)_{n \in \mathbb{N}} \operatorname{com} t_{n} \rightarrow \infty \text { e } \gamma\left(t_{n}\right) \rightarrow q \text {, quando } n \rightarrow \infty\right\} .
$$

Analogamente, definimos o conjunto $\alpha$-limite da trajetória $\gamma$ por

$$
\alpha(\gamma):=\left\{q \in M: \exists\left(t_{n}\right)_{n \in \mathbb{N}} \operatorname{com} t_{n} \rightarrow-\infty \text { e } \gamma\left(t_{n}\right) \rightarrow q \text {, quando } n \rightarrow \infty\right\}
$$

O conjunto $\omega$-limite (respectivamente $\alpha$-limite) da trajetória $\gamma$ também será chamado de conjunto $\omega$-limite da semitrajetória positiva $\gamma^{+} \subset \gamma$ (respectivamente conjunto $\alpha$-limite da semitrajetória negativa $\left.\gamma^{-} \subset \gamma\right)$ e será denotado por $\omega\left(\gamma^{+}\right)$(respectivamente $\alpha\left(\gamma^{-}\right)$). 
Denotemos por $d$ uma métrica em $M$ e sejam $A$ e $B$ subconjuntos de $M$. Recordemos que a distância entre $A$ e $B, d(A, B)$, é definida por

$$
d(A, B)=\inf \{d(p, q): p \in A, q \in B\}
$$

Nas próximas linhas, apresentaremos algumas propriedades do conjunto $\omega$-limite de uma semitrajetória positiva. Propriedades análogas valem para o conjunto $\alpha$-limite de uma semitrajetória negativa.

Proposição 2.2.1 Sejam $X \in \mathfrak{X}(M)$ e $\gamma^{+}=\{\gamma(t): t \geq 0\}$ uma semitrajetória positiva de $X$. Então valem as seguintes propriedades:

(i) $\omega\left(\gamma^{+}\right) \neq \varnothing$;

(ii) $\omega\left(\gamma^{+}\right)$é compacto;

(iii) $\omega\left(\gamma^{+}\right)$é conexo.

\section{Demonstração:}

(i) Seja $\left(t_{n}\right)_{n \in \mathbb{N}}$ uma seqüência de números reais positivos tal que $t_{n} \rightarrow \infty$ quando $n \rightarrow \infty$. Como $M$ é compacta, $\left(\gamma\left(t_{n}\right)\right)_{n \in \mathbb{N}}$ possui uma subseqüência convergente, cujo limite pertence a $\omega\left(\gamma^{+}\right)$. Logo, $\omega\left(\gamma^{+}\right) \neq \varnothing$.

(ii) É suficiente mostrarmos que $\omega\left(\gamma^{+}\right)$é fechado. Assim, suponhamos que exista uma seqüência $\left(p_{n}\right)_{n \in \mathbb{N}}$ em $\omega\left(\gamma^{+}\right)$, com $p_{n} \rightarrow p$ quando $n \rightarrow \infty$. Como $p_{n} \in \omega\left(\gamma^{+}\right)$, podemos tomar $\gamma\left(t_{n}\right)$ e $t_{n}>0$ tais que

$$
d\left(\gamma\left(t_{n}\right), p_{n}\right)<\frac{1}{n}, \text { com } t_{n} \rightarrow \infty \text { quando } n \rightarrow \infty
$$

Como

$$
d\left(\gamma\left(t_{n}\right), p\right)<d\left(\gamma\left(t_{n}\right), p_{n}\right)+d\left(p_{n}, p\right)
$$

concluímos que $\gamma\left(t_{n}\right) \rightarrow p$ quando $n \rightarrow \infty$. Logo $p \in \omega\left(\gamma^{+}\right)$, o que mostra que $\omega\left(\gamma^{+}\right)$é fechado.

(iii) Suponhamos que $\omega\left(\gamma^{+}\right)$não seja um conjunto conexo. Então podemos escrever $\omega\left(\gamma^{+}\right)=A \cup B$, onde $A$ e $B$ são não vazios, fechados (logo, compactos) e $A \cap B=\varnothing$. Seja $\delta=d(A, B)>0$. Como os elementos de $A$ e $B$ pertencem ao conjunto $\omega\left(\gamma^{+}\right)$, existem duas seqüências $\left(t_{n}\right)_{n \in \mathbb{N}}$ e $\left(\tau_{n}\right)_{n \in \mathbb{N}}$ de números reais positivos satisfazendo as seguintes condições: 
(a) $t_{n} \rightarrow \infty$ e $\tau_{n} \rightarrow \infty$, quando $n \rightarrow \infty$;

(b) $d\left(\gamma\left(t_{n}\right), A\right)<\frac{\delta}{4}$ e $d\left(\gamma\left(\tau_{n}\right), B\right)<\frac{\delta}{4}$, para todo $n \in \mathbb{N}$;

(c) $t_{n}<\tau_{n}<t_{n+1}$, para todo $n \in \mathbb{N}$.

Então, para cada $n \in \mathbb{N}$, existe $s_{n}, \operatorname{com} t_{n}<s_{n}<\tau_{n}$, tal que $d\left(\gamma\left(s_{n}\right), A\right) \geq \frac{\delta}{4}$ e $d\left(\gamma\left(s_{n}\right), B\right) \geq$ $\frac{\delta}{4}$. A seqüência $\left(\gamma\left(s_{n}\right)\right)_{n \in \mathbb{N}}$ tem um ponto de acumulação $q \in M$, pois $\gamma^{+} \subset M$ e $M$ é compacta. Claramente $q \in \omega\left(\gamma^{+}\right), d(q, A) \geq \frac{\delta}{4}$ e $d(q, B) \geq \frac{\delta}{4}$, ou seja, $q \in \omega\left(\gamma^{+}\right)$e $q \notin A \cup B$. Esta contradição junto com a suposição de que $\omega\left(\gamma^{+}\right)=A \cup B$ implicam que $\omega\left(\gamma^{+}\right)$é conexo.

Definição 2.2.2 Sejam $\gamma$ e $\widetilde{\gamma}$ trajetórias de $X \in \mathfrak{X}(M)$. Diremos que $\widetilde{\gamma}$ é acompanhada por $\gamma($ ou $\gamma$ acompanha $\widetilde{\gamma})$, se as seguintes condições estiverem satisfeitas:

(1) Existe uma seqüência crescente $\left(t_{n}\right)_{n \in \mathbb{N}}$ de números reais, com $\lim _{n \rightarrow \infty} t_{n}=\infty$;

(2) Para cada $t \in \mathbb{R}, \widetilde{\gamma}(t)=\lim _{n \rightarrow \infty} \gamma\left(t+t_{n}\right)$, onde o limite vale uniformemente em intervalos compactos de $\mathbb{R}$.

A seguir, vamos provar um resultado para campos vetoriais contínuos que garante um tipo de invariância para o conjunto $\omega$-limite. No caso de fluxos contínuos, a invariância do $\omega$ limite segue da dependência contínua das trajetórias com relação às condições iniciais (veja [2, Lema 1.5, p. 48]). Para campos vetoriais contínuos, não temos a dependência contínua das trajetórias com relação às condições iniciais, mas conseguimos garantir que, para cada ponto no $\omega$-limite, existe pelo menos uma trajetória passando por esse ponto e inteiramente contida no conjunto $\omega$-limite. Veja [6, Proposição 2.2].

Proposição 2.2.3 Sejam $X \in \mathfrak{X}(M)$ e $\gamma$ uma trajetória de $X$. Se $p \in \omega(\gamma)$, então existirá uma trajetória $\gamma_{p}$ de $X$ passando por $p$ e acompanhada por $\gamma$. Além disso, $\gamma_{p} \subset \omega(\gamma)$.

\section{Demonstração:}

Seja $\left(t_{n}\right)_{n \in \mathbb{N}}$ uma seqüência crescente de números reais com $t_{n} \rightarrow \infty$ e $\gamma\left(t_{n}\right) \rightarrow p$ quando $n \rightarrow \infty$. Defina $p_{n}=\gamma\left(t_{n}\right)$, para cada $n \in \mathbb{N}$. Então $\gamma_{p_{n}}(t)=\gamma\left(t+t_{n}\right), t \in \mathbb{R}$, é uma trajetória de $X$ passando pelo ponto $p_{n}$. Pelo Teorema 2.1.9, podemos tomar uma subseqüência crescente $\left(t_{n_{k}}\right)_{k \in \mathbb{N}}$ de $\left(t_{n}\right)_{n \in \mathbb{N}}$ e uma trajetória $\gamma_{p}$ de $X$ passando por $p$ e satisfazendo

$$
\gamma_{p}(t)=\lim _{k \rightarrow \infty} \gamma\left(t+t_{n_{k}}\right), t \in \mathbb{R}
$$


onde o limite vale uniformemente em intervalos compactos de $\mathbb{R}$. Isto demonstra a proposição.

Corolário 2.2.4 Nas condições da Proposição 2.2.3, se $\omega(\gamma)$ consistir de um único ponto $p \in M$, então $p$ será um ponto singular de $X e \lim _{t \rightarrow \infty} \gamma(t)=p$.

Definição 2.2.5 Diremos que uma trajetória $\gamma$ de $X \in \mathfrak{X}(M)$ é fracamente $\omega$-recorrente (respectivamente fracamente $\alpha$-recorrente), se existir $p \in \gamma$ tal que $p \in \omega(\gamma)$ (respectivamente $p \in \alpha(\gamma))$. Nestas condições, diremos também que $\gamma$ é fracamente $\omega$-recorrente (respectivamente fracamente $\alpha$-recorrente) no ponto $p$. Uma trajetória será dita fracamente recorrente, se for fracamente $\omega$-recorrente ou fracamente $\alpha$-recorrente. Se, além disso, $\gamma \subset \omega(\gamma)$ (respectivamente $\gamma \subset \alpha(\gamma)$ ), diremos que $\gamma$ é $\omega$-recorrente (respectivamente $\alpha$-recorrente). Finalmente, diremos que $\gamma$ é recorrente, se for $\omega$-recorrente ou $\alpha$-recorrente.

Um ponto singular e uma trajetória periódica são trajetórias recorrentes. Em cada um destes casos, dizemos que a trajetória é recorrente trivial ou fracamente recorrente trivial.

A seguir, apresentaremos um exemplo de um campo vetorial contínuo no toro $T^{2}$ onde toda trajetória é recorrente não-trivial.

Exemplo 2.2.6 (Veja [18, p. 13]) Seja $\varphi: \mathbb{R}^{2} \rightarrow T^{2} \subset \mathbb{R}^{3}$ definida por

$$
\varphi(u, v)=((2+\cos 2 \pi v) \cos 2 \pi u,(2+\cos 2 \pi v) \operatorname{sen} 2 \pi u, \operatorname{sen} 2 \pi v) .
$$

O conjunto

$$
T^{2}=\left\{(x, y, z) \in \mathbb{R}^{3}:\left[\left(x^{2}+y^{2}\right)^{\frac{1}{2}}-2\right]^{2}+z^{2}=1\right\}
$$

é o toro obtido pela rotação da circunferência

$$
\left\{(x, y) \in \mathbb{R}^{2}:(x-2)^{2}+y^{2}=1\right\}
$$

em torno do eixo $z$ (veja Figura 2.1).

A aplicação $\varphi$ é um difeomorfismo local que leva as retas horizontais de $\mathbb{R}^{2}$ em paralelos de $T^{2}$, as retas verticais em meridianos e o quadrado $[0,1] \times[0,1]$ sobre $T^{2}$. Além disso, $\varphi(u, v)=\varphi(\widetilde{u}, \widetilde{v})$ se, e somente se, $u-\widetilde{u}=m$ e $v-\widetilde{v}=n$, onde $m, n \in \mathbb{Z}$. 


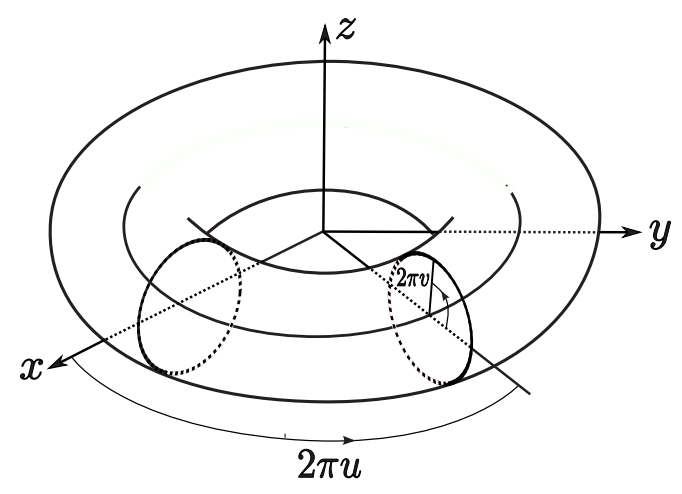

Figura 2.1: O toro $T^{2}$.

Para cada $a \in \mathbb{R}$, consideremos o campo vetorial $X^{a}: \mathbb{R}^{2} \rightarrow \mathbb{R}^{2}$ dado por

$$
X^{a}(u, v)=(1, a)
$$

Notemos que $Y^{a}=\varphi_{*} X^{a}=d \varphi \circ X^{a} \circ \varphi^{-1}$ está bem definido e é um campo vetorial de classe $C^{\infty}$ no toro $T^{2}$. As trajetórias de $Y^{a}$ são as imagens por $\varphi$ das trajetórias de $X^{a}$, sendo estas as retas de inclinação $a$ em $\mathbb{R}^{2}$.

Mostraremos que, para a racional, toda trajetória de $Y^{a}$ é periódica e, para $a$ irracional, toda trajetória de $Y^{a}$ é densa em $T^{2}$.

Para cada $c \in \mathbb{R}$, denotemos por $\Delta_{c}$ a reta de $\mathbb{R}^{2}$ que passa pelo ponto $(0, c)$ e tem inclinação $a$, isto é, $\Delta_{c}=\{(u, c+a u): u \in \mathbb{R}\}$. Se $a$ for racional, a trajetória $\varphi\left(\Delta_{c}\right)$ de $Y^{a}$ será periódica, para todo $c \in \mathbb{R}$. De fato, se $a=n / m$, onde $m, n \in \mathbb{Z}$, então $(m, c+(n / m) m) \in \Delta_{c}$ e $\varphi(m, c+n)=\varphi(0, c)$.

Suponhamos, agora, que a seja irracional e fixemos $\bar{c} \in \mathbb{R}$. Afirmamos que o conjunto $C=\left\{c \in \mathbb{R}: \varphi\left(\Delta_{c}\right)=\varphi\left(\Delta_{\bar{c}}\right)\right\}$ é denso em $\mathbb{R}$. Supondo que a nossa afirmação seja válida, então $\cup_{c \in C} \Delta_{c}$ é denso em $\mathbb{R}^{2}$ e, portanto, $\varphi\left(\Delta_{\bar{c}}\right)=\varphi\left(\cup_{c \in C} \Delta_{c}\right)$ é denso em $T^{2}$. Logo, basta provarmos a nossa afirmação.

Para mostrarmos que $C$ é denso em $\mathbb{R}$ é suficiente mostrarmos que o conjunto $G=$ $\{m a+n: m, n \in \mathbb{Z}\}$ é denso em $\mathbb{R}$, pois $c \in C$ se, e somente se, $c-\bar{c} \in G$. Como $G$ é um subgrupo de $\mathbb{R}$ com a operação soma, então ou $G$ é denso ou $G$ é discreto. Resta, assim, mostrarmos que $G$ não é discreto. Com efeito, para cada $m \in \mathbb{Z}$, existe $n \in \mathbb{Z}$ tal que $u_{m}=m a+n$ pertence ao intervalo $[0,1]$. A seqüência $\left(u_{m}\right)_{m \in \mathbb{N}}$ tem um ponto de acumulação e, como a é irracional, seus elementos são distintos. Logo, $G$ é denso. 
O campo $Y^{a}$ é chamado de campo racional ou irracional em $T^{2}$ conforme a seja racional ou não. Se $a$ for racional, o conjunto $\omega$-limite de qualquer trajetória será ela própria. Se $a$ for irracional, o conjunto $\omega$-limite de qualquer trajetória será todo o toro $T^{2}$.

\subsection{Campos vetoriais definidos por um recobrimento du- plo orientado.}

Nesta seção, apresentamos a definição de levantamento de um campo vetorial contínuo por um recobrimento duplo orientado entre variedades compactas bidimensionais. A teoria de espaço de recobrimento duplo orientado pode ser encontrada em [14]. O resultado final desta seção diz que, se conhecermos o conjunto $\omega$-limite de uma trajetória do campo levantado, então também conheceremos o conjunto $\omega$-limite da trajetória projeção (Lema 2.3.7).

Definição 2.3.1 Sejam $M$ e $\widetilde{M}$ variedades compactas bidimensionais (com ou sem bordo). Um recobrimento duplo orientado é uma aplicação $\pi: \widetilde{M} \rightarrow M$ de classe $C^{r}, r \geq 1$, com as seguintes propriedades:

(1) $\widetilde{M}$ é uma variedade orientada e $M$ é uma variedade não-orientável;

(2) $\pi$ é um difeomorfismo local;

(3) Para cada $p \in M$, a imagem inversa $\pi^{-1}(p)$ contém exatamente dois pontos;

Segue da definição acima que, para todo aberto $U \subset M, \pi^{-1}(U)=\widetilde{U}_{1} \cup \widetilde{U}_{2}$ é união disjunta de dois abertos de $\widetilde{M}$, cada um dos quais se aplica, por $\pi$, difeomorficamente sobre $U$.

Às vezes se diz, por abuso de linguagem, que $\widetilde{M}$ é um recobrimento duplo orientado de $M$.

No que segue, mostraremos como são caracterizados os recobrimentos duplos orientáveis.

Dada uma variedade compacta bidimensional $\widetilde{M}$ (com ou sem bordo). Denotemos por $i d: \widetilde{M} \rightarrow \widetilde{M}$ a aplicação identidade de $\widetilde{M}$.

Seja $\alpha: \widetilde{M} \rightarrow \widetilde{M}$ uma involução (isto é, $\alpha \circ \alpha=i d$ ) de classe $C^{r}$ que inverte a orientação de $\widetilde{M}$ e não possui pontos fixos. Então $\{i d, \alpha\}$ é um grupo propriamente descontínuo de 
difeomorfismos de $\widetilde{M}$. Indicaremos por $\widetilde{M} / \alpha$ a variedade quociente. Pela Proposição 3 de [14, p. 193], a aplicação quociente $\Pi: \widetilde{M} \rightarrow \widetilde{M} / \alpha$ é um recobrimento duplo orientado. Reciprocamente, o Corolário da Proposição 6 de [14, p. 198] diz que todo recobrimento duplo orientado é essencialmente obtido dessa maneira. Mais precisamente:

Corolário 2.3.2 Seja $\pi: \widetilde{M} \rightarrow M$ um recobrimento duplo orientado. Existe uma única involução $\alpha: \widetilde{M} \rightarrow \widetilde{M}$ de classe $C^{r}$ que inverte a orientação de $\widetilde{M}$ tal que $\pi \circ \alpha=\pi$. $A$ involução $\alpha$ não tem pontos fixos. Existe um único difeomorfismo $\xi: \widetilde{M} / \alpha \rightarrow M$ tal que $\pi=\xi \circ \Pi$, onde $\Pi: \widetilde{M} \rightarrow \widetilde{M} / \alpha$ é a aplicação quociente.

A seguir, mostraremos que a esfera $S^{2}$ é um recobrimento duplo orientado do plano projetivo $P^{2}$, que o toro $T^{2}$ é um recobrimento duplo orientado da garrafa de Klein $K^{2}$ e que o cilindro é um recobrimento duplo orientado da faixa de Möbius. Estes exemplos foram extraídos de [14].

Exemplo 2.3.3 Consideremos a aplicação antípoda da esfera unitária $S^{2}$,

$$
\alpha: S^{2} \rightarrow S^{2}, \alpha(x)=-x
$$

a qual não tem pontos fixos. Como $\alpha$ é uma involução que inverte a orientação de $S^{2}$, então a aplicação quociente $\Pi: S^{2} \rightarrow S^{2} / \alpha$ é um recobrimento duplo orientado de $S^{2}$ no plano projetivo $P^{2}=S^{2} / \alpha$.

Exemplo 2.3.4 Seja $T^{2}=S^{1} \times S^{1}$, onde $S^{1}=\{z \in \mathbb{C}:|z|=1\}$. Consideremos a aplicação $\alpha: T^{2} \rightarrow T^{2}$ definida por

$$
\alpha(z, w)=(\bar{z},-w), z, w \in S^{1}
$$

onde $\bar{z}$ é o número complexo conjugado de $z$. Não é difícil ver que $\alpha$ é uma involução de classe $C^{\infty}$. Além disso, $\alpha$ inverte a orientação de $T^{2}$ e não tem pontos fixos.

A variedade quociente $T^{2} / \alpha$ é difeomorfa à garrafa de Klein $K^{2}$. Assim, a aplicação quociente $\Pi: T^{2} \rightarrow T^{2} / \alpha$ representa o toro como recobrimento duplo orientado da garrafa de Klein.

Exemplo 2.3.5 A aplicação $\alpha: S^{1} \times[0,1] \rightarrow S^{1} \times[0,1]$ definida por

$$
\alpha(z, t)=(-z,-t),
$$


é uma involução que inverte a orientação de $S^{1} \times[0,1]$ e não possui pontos fixos. Logo, a aplicação quociente $\Pi: S^{1} \times[0,1] \rightarrow\left(S^{1} \times[0,1]\right) / \alpha$ é um recobrimento duplo orientado do cilindro $S^{1} \times[0,1]$ na faixa de Möbius $\left(S^{1} \times[0,1]\right) / \alpha$.

Nosso próximo passo será definir campos vetoriais contínuos a partir de um recobrimento duplo orientado.

Seja $\pi: \widetilde{M} \rightarrow M$ um recobrimento duplo orientado. Dado um campo vetorial contínuo $X$ em $M$, podemos definir um campo vetorial contínuo $\pi^{*}(X)$ em $\widetilde{M}$, denominado levantamento de $X$, por

$$
\pi^{*}(X)(p)=\left(d \pi_{p}\right)^{-1} \cdot X(\pi(p)), p \in \widetilde{M}
$$

Fixemos $p \in M$ e consideremos $\pi^{-1}(p)=\{\widetilde{p}, \hat{p}\}$. Seja $\gamma: \mathbb{R} \rightarrow M$ uma curva integral de $X$ passando por $p$. Pelo Teorema Fundamental de Levantamento (veja [14, Proposição 4, p. 153] e [14, Proposição 2', p. 119]), existe um único caminho de classe $C^{1} \widetilde{\gamma}: \mathbb{R} \rightarrow \widetilde{M}$ satisfazendo $\widetilde{\gamma}(0)=\widetilde{p}$ e $\pi \circ \widetilde{\gamma}=\gamma$.

Derivando ambos os membros da igualdade $\pi \circ \widetilde{\gamma}=\gamma$ com relação a variável $t$, obtemos

$$
d \pi_{\widetilde{\gamma}(t)} \cdot \widetilde{\gamma}^{\prime}(t)=\gamma^{\prime}(t)
$$

Como $d \pi_{\widetilde{\gamma}(t)}$ é um isomorfismo e $\gamma^{\prime}(t)=X(\gamma(t))$, vale

$$
\widetilde{\gamma}^{\prime}(t)=\left(d \pi_{\widetilde{\gamma}(t)}\right)^{-1} \cdot X(\gamma(t))
$$

ou seja, $\widetilde{\gamma}^{\prime}(t)=\pi^{*}(X)(\widetilde{\gamma}(t))$. Portanto, $\widetilde{\gamma}$ é uma curva integral de $\pi^{*}(X)$ passando por $\widetilde{p}$.

Analogamente, existe uma única curva integral $\hat{\gamma}: \mathbb{R} \rightarrow \widetilde{M}$ de $\pi^{*}(X)$ passando por $\hat{p}$ e satisfazendo $\pi \circ \hat{\gamma}=\gamma$.

Disto segue que, ou $\pi^{-1}(\gamma)$ é a união de duas trajetórias disjuntas de $\pi^{*}(X)$, digamos $\widetilde{\gamma}$ e $\hat{\gamma}$, ou $\pi^{-1}(\gamma)$ é igual a $\widetilde{\gamma}$ e, neste caso, $\hat{\gamma}$ é uma reparametrização da curva $\widetilde{\gamma}$.

Resumidamente, concluímos que a projeção $\gamma=\pi \circ \widetilde{\gamma}$ de uma trajetória $\widetilde{\gamma}$ de $\pi^{*}(X)$ é uma trajetória de $X$. Reciprocamente, toda trajetória de $X$ é a projeção de, no máximo, duas trajetórias de $\pi^{*}(X)$.

Lema 2.3.6 Sejam $\pi: \widetilde{M} \rightarrow M$ um recobrimento duplo orientado e $\pi^{*}(X) \in \mathfrak{X}(\widetilde{M})$ o levantamento de $X \in \mathfrak{X}(M)$. Sejam $\widetilde{\gamma}$ uma trajetória de $\pi^{*}(X)$ e $\gamma=\pi \circ \widetilde{\gamma}$ a trajetória 
correspondente de $X$. Então as seguintes afirmações se verificam:

(i) $\gamma$ será um ponto singular, e somente se, $\widetilde{\gamma}$ for um ponto singular;

(ii) $\gamma$ será uma trajetória injetiva se, e somente se, $\widetilde{\gamma}$ for injetiva;

(iii) $\gamma$ será uma trajetória periódica se, e somente se, $\widetilde{\gamma}$ for periódica.

\section{Demonstração:}

Imediata.

O lema seguinte desempenhará um papel importante no próximo capítulo.

Lema 2.3.7 Nas condições do Lema 2.3.6, temos $\pi(\omega(\widetilde{\gamma}))=\omega(\gamma)$.

\section{Demonstração:}

Seja $\widetilde{p} \in \omega(\widetilde{\gamma})$. Tomemos uma seqüência $\left(t_{n}\right)_{n \in \mathbb{N}}$ de números reais tal que $t_{n} \rightarrow \infty \mathrm{e}$ $\widetilde{\gamma}\left(t_{n}\right) \rightarrow \widetilde{p}$, quando $n \rightarrow \infty$. Sendo $\pi$ contínua, temos

$$
\lim _{n \rightarrow \infty} \pi\left(\widetilde{\gamma}\left(t_{n}\right)\right)=\pi(\widetilde{p})
$$

ou seja,

$$
\lim _{n \rightarrow \infty} \gamma\left(t_{n}\right)=\pi(\widetilde{p}) \in \pi(\omega(\widetilde{\gamma}))
$$

Logo, $\pi(\omega(\widetilde{\gamma})) \subset \omega(\gamma)$.

Reciprocamente, seja $p \in \omega(\gamma)$. Então existe uma seqüência $\left(s_{n}\right)_{n \in \mathbb{N}}$ de números reais tal que $s_{n} \rightarrow \infty$ e $\gamma\left(s_{n}\right) \rightarrow p$, quando $n \rightarrow \infty$.

Consideremos um aberto $U \subset M$ tal que $p \in U$ e $\pi^{-1}(U)=\widetilde{U}_{1} \cup \widetilde{U}_{2}$, onde $\widetilde{U}_{1}$ e $\widetilde{U}_{2}$ são abertos disjuntos de $\widetilde{M}$ e $\left.\pi\right|_{\widetilde{U}_{i}}: \widetilde{U}_{i} \rightarrow U$ é um difeomorfismo, $i=1,2$. Podemos supor que $\gamma\left(s_{n}\right) \in U$, para todo $n \in \mathbb{N}$. Daí, $\left(\widetilde{\gamma}\left(s_{n}\right)\right)_{n \in \mathbb{N}}$ tem uma subseqüência, que ainda denominaremos por $\left(\widetilde{\gamma}\left(s_{n}\right)\right)_{n \in \mathbb{N}}$, a qual está contida em $\widetilde{U}_{1}$ ou em $\widetilde{U}_{2}$.

Para fixar as idéias, suponhamos que $\left(\widetilde{\gamma}\left(s_{n}\right)\right)_{n \in \mathbb{N}}$ esteja contida em $\widetilde{U}_{1}$. Como $\left(\left.\pi\right|_{\widetilde{U}_{1}}\right)^{-1}$ : $U \rightarrow \widetilde{U}_{1}$ é contínua e $\pi\left(\widetilde{\gamma}\left(s_{n}\right)\right)=\gamma\left(s_{n}\right) \rightarrow p$, quando $n \rightarrow \infty$, então

$$
\lim _{n \rightarrow \infty} \widetilde{\gamma}\left(s_{n}\right)=\left(\left.\pi\right|_{\widetilde{U}_{1}}\right)^{-1}(p)
$$

Daí, $\pi^{-1}(p) \cap \widetilde{U}_{1} \in \omega(\widetilde{\gamma})$. Logo $p \in \pi(\omega(\widetilde{\gamma}))$ e, portanto, $\omega(\gamma) \subset \pi(\omega(\widetilde{\gamma}))$. Isto conclui a demonstração do lema. 
Observação 2.3.8 Pelo Lema 2.3.7, $\omega(\widetilde{\gamma}) \subset \pi^{-1}(\omega(\gamma))$. Mas não é sempre verdade que $\pi^{-1}(\omega(\gamma))=\omega(\widetilde{\gamma})$, pois se $\hat{\gamma}$ for a trajetória de $\pi^{*}(X)$ disjunta de $\widetilde{\gamma}$ em $\pi^{-1}(\gamma)$, pode-se provar que $\pi^{-1}(\omega(\gamma))=\omega(\widetilde{\gamma}) \cup \omega(\hat{\gamma})$. Um exemplo em que este caso ocorre é quando $\gamma$ é uma trajetória periódica homotópica a um ponto. Neste caso, as trajetórias $\widetilde{\gamma}$ e $\hat{\gamma}$ são periódicas, disjuntas e homotópicas a um ponto. Daí,

$$
\pi^{-1}(\omega(\gamma))=\pi^{-1}(\gamma)=\widetilde{\gamma} \cup \hat{\gamma}=\omega(\widetilde{\gamma}) \cup \omega(\hat{\gamma})
$$




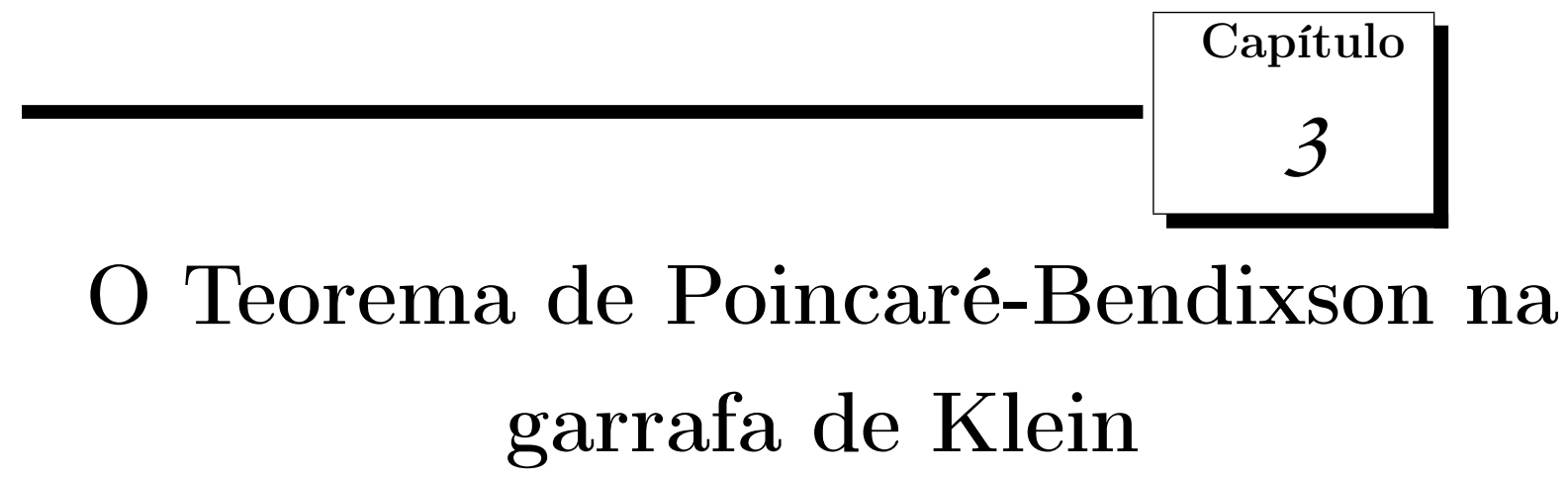

Este capítulo está divido em duas partes. Na primeira parte, tratamos dos aspectos topológicos da garrafa de Klein $K^{2}$, os quais apresentamos em diversos resultados auxiliares. Um destes resultados nos diz que o complementar de um certo tipo de curva fechada simples em $K^{2}$ é um cilindro (Lema 3.1.9). Um outro resultado nos dá informações sobre a existência dessas curvas em $K^{2}$ (Lema 3.1.10).

Na segunda parte deste capítulo, apresentamos uma versão do Teorema de PoincaréBendixson para campos vetoriais contínuos na garrafa de Klein $K^{2}$ em duas situações. Assumimos que o campo vetorial contínuo tem um número finito de singularidades e consideramos uma trajetória injetiva. Então descrevemos o conjunto $\omega$-limite desta trajetória no caso em que ela é fracamente $\omega$-recorrente (Teorema 3.2.6) e no caso em que ela não é fracamente $\omega$-recorrente (Teorema 3.2.10). Como conseqüência, concluímos que um campo vetorial contínuo em $K^{2}$ não possui trajetória injetiva que seja $\omega$-recorrente.

Os resultados principais deste capítulo podem ser encontrados em [6].

\subsection{Aspectos topológicos}

O objetivo desta seção é tratarmos dos aspectos topológicos da garrafa de Klein $K^{2}$. Para isto, precisaremos do conhecimento de alguns resultados clássicos de topologia algébrica. Tais resultados foram extraídos, sem demonstrações, de [16]. 
Primeiramente, apresentamos um método para se construir variedades compactas bidimensionais a partir de outras.

Sejam $M_{1}$ e $M_{2}$ variedades compactas bidimensionais disjuntas. Considere a seguinte "cirurgia": remova dois pequenos discos abertos (isto é, homeomorfos a $\left\{(x, y) \in \mathbb{R}^{2}\right.$ : $\left.\left.x^{2}+y^{2}<1\right\}\right)$ em cada uma das variedades e, em seguida, "cole" ambas as variedades ao longo dos bordos que resultaram da remoção dos discos. O espaço topológico assim formado é uma variedade compacta bidimensional que é chamada de soma conexa de $M_{1}$ e $M_{2}$ e denotada por $M_{1} \# M_{2}$.

Uma descrição mais rigorosa da soma conexa de $M_{1}$ e $M_{2}$ é esta: sejam $D_{1} \subset M_{1}$ e $D_{2} \subset M_{2}$ discos fechados (isto é, homeomorfos a $\left\{(x, y) \in \mathbb{R}^{2}: x^{2}+y^{2} \leq 1\right\}$ ). Denotemos por $M_{i}^{c}$ o complementar do interior de $D_{i}$ em $M_{i}, i=1,2$. Se $h: \partial M_{1}^{c} \rightarrow \partial M_{2}^{c}$ for um homeomorfismo do bordo de $M_{1}^{c}$ no bordo de $M_{2}^{c}$, então $M_{1} \# M_{2}$ é o espaço quociente de $M_{1}^{c} \cup M_{2}^{c}$ obtido identificando-se os pontos $p \operatorname{com} h(p)$, para todo $p \in \partial M_{1}^{c}$. O tipo de topologia da variedade $M_{1} \# M_{2}$ não depende da escolha dos discos $D_{1}$ e $D_{2}$ nem da escolha do homeomorfismo $h$.

Observamos que, se $M_{1}$ e $M_{2}$ forem variedades orientáveis, então $M_{1} \# M_{2}$ também será orientável, enquanto que $M_{1} \# M_{2}$ não será orientável, se $M_{1}$ ou $M_{2}$ não o for. Por exemplo, a soma conexa de dois planos projetivos é homeomorfa à garrafa de Klein $K^{2}$. Na Figura 3.1, temos a soma conexa de dois toros $T^{2}$.

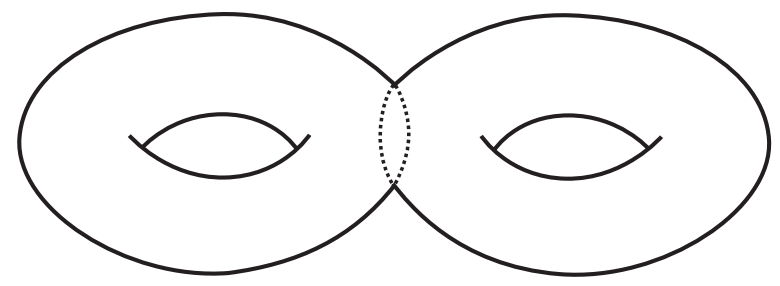

Figura 3.1: $T^{2} \# T^{2}$.

Teorema 3.1.1 [16, Teorema 5.1, p. 10] Toda variedade bidimensional compacta é homeomorfa ou à esfera, ou à soma conexa de toros ou à soma conexa de planos projetivos.

O teorema acima mostra que não precisamos considerar variedades que são somas conexas de toros e planos projetivos. 
Sabemos, então, que toda variedade bidimensional compacta é homeomorfa ou à esfera, ou à soma conexa de toros ou à soma conexa de planos projetivos. Entretanto não sabemos se todas estas variedades são topologicamente distintas. Para mostrar que esta última afirmação é verdadeira, apresentaremos um invariante conhecido como característica de Euler. Antes, porém, precisaremos de um resultado auxiliar apresentado a seguir.

Dada uma variedade compacta bidimensional $M$. Uma triangulação de $M$ é uma família finita de triângulos curvilíneos (imagens difeomorfas de triângulos do plano) que cobrem $M$, de tal modo que dois quaisquer deles, ou não se interceptam, ou têm somente um vértice em comum, ou então têm precisamente um lado em comum.

Uma variedade compacta bidimensional $M$ sempre pode ser triangulada (veja [22]). Admitiremos este resultado, pois sua demonstração não se identifica com os objetivos deste trabalho.

Definição 3.1.2 A característica de Euler de uma variedade compacta bidimensional $M$ é dada pela fórmula

$$
\chi(M)=v-a+f
$$

onde $v$, a e $f$ são respectivamente o número de vértices, arestas e faces de uma triangulação de $M$.

Podemos provar que o número $\chi(M)$ é um invariante topológico, isto é, não depende da triangulação escolhida para $M$, embora $v$, a e $f$ dependam. Assim, o número $\chi(M)$ identifica $M$ a menos de homeomorfismos.

Exemplos 3.1.3 (Veja [16, Exemplo 8.1, p. 29])

(a) $\chi\left(S^{2}\right)=2$.

(b) $\chi\left(T^{2}\right)=0$.

(c) $\chi\left(P^{2}\right)=1$.

Proposição 3.1.4 [16, Proposição 8.1, p. 33] Sejam $M_{1}$ e $M_{2}$ variedades compactas bidimensionais disjuntas. Então

$$
\chi\left(M_{1} \# M_{2}\right)=\chi\left(M_{1}\right)+\chi\left(M_{2}\right)-2 .
$$


A proposição anterior nos permite mostrar que a característica de Euler de uma variedade compacta bidimensional, formada pela soma conexa de

(a) $n$ toros, é $2-2 n$;

(b) $n$ planos projetivos, é $2-n$;

(c) um plano projetivo e $n$ toros, é $1-2 n$;

(d) uma garrafa de Klein e $n$ toros, é $-2 n$.

Por exemplo,

$$
\chi(T \# T)=-2, \text { pois } \chi(T)=0 .
$$

Pelo que foi exposto acima, percebemos que a característica de Euler de uma variedade orientável é sempre par, enquanto que a característica de Euler de uma variedade nãoorientável pode ser par ou ímpar.

Assumindo a invariância topológica da característica de Euler e o Teorema 3.1.1, temos o seguinte resultado importante.

Teorema 3.1.5 (Teorema de Classificação de Variedades) [16, Teorema 8.2, p. 33] Sejam $M_{1}$ e $M_{2}$ variedades compactas bidimesionais. Então, $M_{1}$ e $M_{2}$ serão homeomorfas se, e somente se, suas características de Euler forem iguais e ambas forem orientáveis ou não-orientáveis.

O teorema acima reduz o problema de classificação das variedades compactas bidimensionais à determinação da orientabilidade e da característica de Euler, ambos problemas solúveis. Logo, o Teorema 3.1.1 caracteriza as possíveis variedades compactas bidimensionais.

Uma variedade compacta bidimensional que é soma conexa de $n$ toros ou $n$ planos projetivos é dita ser de gênero $n$. Em particular, a esfera é dita ser de gênero 0 .

A seguinte relação entre o gênero $g$ e a característica $\chi$ de uma variedade compacta bidimensional vale:

$$
g= \begin{cases}\frac{1}{2}(2-\chi), & \text { no caso orientável } \\ 2-\chi, & \text { no caso não-orientável. }\end{cases}
$$


Vamos prosseguir com nossa exposição a fim de apresentarmos um teorema de classificação para as variedades compactas bidimensionais com bordo.

Seja $M$ uma variedade compacta bidimensional com bordo e suponhamos que este bordo tenha $k$ componentes, $k \geq 1$. Cada componente do bordo é homeomorfa a um círculo. Se tomarmos $k$ discos fechados e colarmos o bordo do i-ésimo disco na i-ésima componente do bordo de $M$ obteremos uma variedade compacta bidimensional $M^{*}$. O tipo de topologia da variedade $M^{*}$ depende somente do tipo de topologia de $M$. O que não é óbvio é que o tipo de topologia de uma variedade com bordo $M$ depende somente do número de componentes do bordo e do tipo de topologia da variedade $M^{*}$, obtida por colar um disco fechado em cada uma das componentes do bordo. Mas isto é verdadeiro como mostra o teorema abaixo.

Teorema 3.1.6 [16, Teorema 10.1, p. 37] Sejam $M_{1}$ e $M_{2}$ variedades compactas bidimensionais com bordo. Suponhamos que o seus bordos tenham o mesmo número de componentes. Então $M_{1}$ e $M_{2}$ são homeomorfas se, e somente se, as variedades $M_{1}^{*}$ e $M_{2}^{*}$, obtidas por colar um disco fechado em cada uma das componentes do bordo, são homeomorfas.

A característica de Euler de uma variedade compacta bidimensional com bordo triangulada é definida exatamente da mesma forma para o caso de uma variedade sem bordo. Usando a característica de Euler, daremos uma classificação completa das variedades compactas bidimesionais com bordo.

Se começarmos com uma variedade compacta bidimesional $M^{*}$ (sem bordo) e removermos dela o interior de $k$ triângulos disjuntos, obteremos uma variedade compacta bidimensional com bordo $M$ de forma que

$$
\chi(M)=\chi\left(M^{*}\right)-k .
$$

Assim, a característica de Euler de $M$ é unicamente determinada pela característica de Euler de $M^{*}$ e vice versa. Usando esta relação, juntamente com o Teorema 3.1.1, obtemos todas as variedades compactas bidimensionais cujo o bordo tem $k$ componentes. Também é verdade que $M$ e $M^{*}$ são ambas orientáveis ou ambas não-orientáveis. Logo, usando o Teorema 3.1.6 e o Teorema 3.1.1, prova-se o seguinte resultado.

Teorema 3.1.7 [16, Teorema 11.1, p. 42] Duas variedades compactas com bordo serão homeomorfas se, e somente se, elas tiverem o mesmo número de componentes de bordo, forem 
ambas orientáveis ou não-orientavéis e tiverem a mesma característica de Euler.

Uma propriedade importante da característica de Euler é a seguinte:

$$
\chi\left(M_{1} \cup M_{2}\right)=\chi\left(M_{1}\right)+\chi\left(M_{2}\right)-\chi\left(M_{1} \cap M_{2}\right),
$$

desde que $M_{1}, M_{2}$ e $M_{1} \cap M_{2}$ sejam variedades compactas bidimensionais (com ou sem bordo). Para o leitor interessado, nos referimos a [24] para mais detalhes.

A seguir, definiremos os conceitos de curva de um lado e curva de dois lados.

Definição 3.1.8 Consideremos uma curva fechada simples $C$ em uma variedade compacta bidimensional $M$. Diremos que $C$ é de dois lados, se ela possuir uma vizinhança homeomorfa a um cilindro. Caso contrário, diremos que $C$ é de um lado.

Observamos que uma curva de um lado possui uma vizinhança homeomorfa a uma faixa de Möbius.

As curvas fechadas simples na esfera $S^{2}$ ou no toro $T^{2}$ são exemplos de curvas de dois lados.

Dadas uma curva de dois lados $C$ sobre uma variedade compacta bidimensional $M$ e uma vizinhança $N(C)$ de $C$ homeomorfa a um cilindro, então $N(C) \backslash C$ tem duas componentes conexas. Definimos os dois lados de $C$ como sendo estas componentes conexas.

Na seqüência, iremos tratar de curvas fechadas simples na garrafa de Klein $K^{2}$.

O lema seguinte diz que se o complementar de uma curva de dois lados na garrafa de Klein $K^{2}$ for conexo, então ele será um cilindro. Uma prova deste resultado foi dada em [9], usando-se o Teorema do Índice de Poincaré-Hopf (veja [21]). A prova que apresentaremos a seguir é diferente daquela dada em [9], pois usa apenas propriedades topológicas de $K^{2}$.

Lema 3.1.9 Seja $C$ uma curva fechada simples na garrafa de Klein $K^{2}$. Se $C$ for de dois lados e $K^{2} \backslash C$ for conexo, então $K^{2} \backslash C$ será um cilindro.

\section{Demonstração:}


Sejam $V_{0}$ e $V_{1}$ vizinhanças de $C$ homeomorfas a um cilindro e tais que $\overline{V_{0}} \subset V_{1}$. Por (3.3), temos

$$
\chi\left(K^{2}\right)=\chi\left(K^{2} \backslash V_{0}\right)+\chi\left(\overline{V_{1}}\right)-\chi\left(\left(K^{2} \backslash V_{0}\right) \cap \overline{V_{1}}\right) .
$$

Como $\chi\left(K^{2}\right)=0, \chi\left(\overline{V_{1}}\right)=\chi\left(S^{1} \times[0,1]\right)=0$ e $\chi\left(\left(K^{2} \backslash V_{0}\right) \cap \overline{V_{1}}\right)=\chi\left(\left(S^{1} \times[0,1]\right) \cup\left(S^{1} \times\right.\right.$ $[0,1]))=0$, então $\chi\left(K^{2} \backslash V_{0}\right)=0$. Daí, sendo $K^{2} \backslash V_{0}$ uma variedade compacta cujo bordo consiste de dois círculos, a igualdade (3.2), juntamente com o Teorema de Classificação de Variedades, implicam que $K^{2} \backslash V_{0}$ é um cilindro, ou seja, $K^{2} \backslash C$ é um cilindro. Isto completa a prova.

O próximo resultado nos diz que a garrafa de Klein não admite três curvas fechadas simples e disjuntas que sejam de um lado só.

Lema 3.1.10 $S e C_{1}, C_{2}$ e $C_{3}$ forem três curvas fechadas simples e disjuntas na garrafa de Klein $K^{2}$, então pelo menos uma das curvas será de dois lados.

\section{Demonstração:}

Mostraremos que se $C_{1}$ e $C_{2}$ forem curvas de um lado, então $C_{3}$ será necessariamente uma curva de dois lados.

Seja $N\left(C_{1}\right)$ uma vizinhança de $C_{1}$ em $K^{2}$ homeomorfa a uma faixa de Möbius e tal que $N\left(C_{1}\right) \cap C_{2}=\varnothing$. Segue, do Teorema de Classificação de Variedades, que a variedade obtida por colar um disco fechado no bordo de $K^{2} \backslash N\left(C_{1}\right)$ é o plano projetivo $P^{2}$. Como $C_{2}$ é uma curva de um lado em $P^{2}$, então podemos considerar uma vizinhança $N\left(C_{2}\right)$ de $C_{2}$ em $P^{2}$ homeomorfa a uma faixa de Möbius e tal que $N\left(C_{2}\right) \cap C_{3}=\varnothing$. Usando novamente o Teorema de Classificação de Variedades, concluímos que a variedade obtida por colarmos um disco fechado no bordo de $P^{2} \backslash N\left(C_{2}\right)$ é a esfera $S^{2}$. Como todas as curvas fechadas em $S^{2}$ são de dois lados e $C_{3}$ está contida em $S^{2}$, então $C_{3}$ é necessariamente de dois lados. O lema está demonstrado.

Para finalizarmos esta seção, apresentamos o grupo fundamental de $K^{2}$ que é denotado por $\pi_{1}\left(K^{2}\right)$.

Seja $G$ o grupo gerado pelos homeomorfismos $\alpha: \mathbb{R}^{2} \rightarrow \mathbb{R}^{2}, \alpha(u, v)=(u+1, v)$, e $\beta: \mathbb{R}^{2} \rightarrow \mathbb{R}^{2}, \beta(u, v)=(-u, v+1)$. Como $\alpha \beta=\beta \alpha^{-1}$, os elementos de $G$ podem ser escritos sob a forma $\beta^{m} \alpha^{n}$, onde $m, n \in \mathbb{Z}$. Afirmamos que $G$ é um grupo propriamente descontínuo 
de homeomorfismos de $\mathbb{R}^{2}$ e o espaço quociente $\mathbb{R}^{2} / G$ é a garrafa de Klein $K^{2}$. Assim, o grupo fundamental de $K^{2}$ é isomorfo a $G$ (veja [14, Exemplo 13, p. 168]).

A garrafa de Klein pode ser visualizada como o espaço obtido a partir de um quadrado pela identificação dos lados opostos como mostra a Figura 3.2.

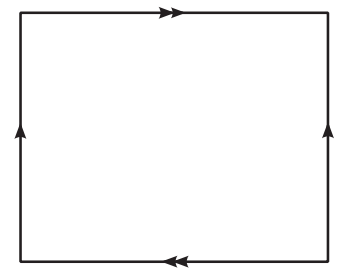

Figura 3.2: A garrafa de Klein $K^{2}$.

Uma curva fechada simples que não é homotópica a um ponto em $K^{2}$ representa um dos seguinte elementos de $\pi_{1}\left(K^{2}\right)$ :

$$
\alpha, \alpha^{-1}, \beta^{2}, \beta^{-2} \text { ou } \beta^{-1} \alpha^{n}, n \in \mathbb{Z}
$$

A prova deste resultado pode ser encontrada em [7, Lema 2.3, p. 138].

As figuras abaixo mostram como essas classes de homotopia podem ser representadas por curvas fechadas simples.

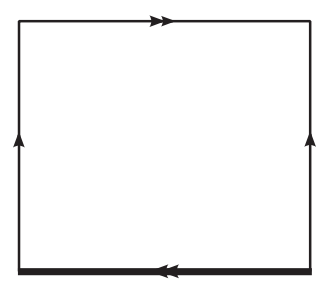

(a) $\alpha$

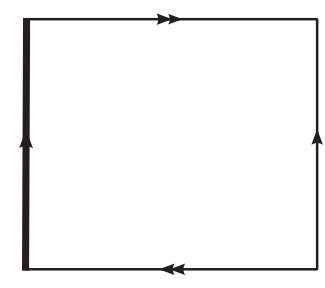

(b) $\beta$

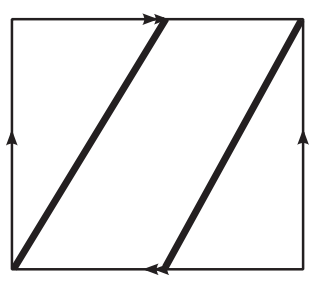

(c) $\beta^{2}$

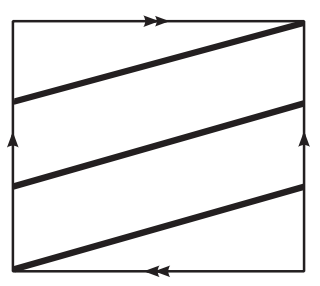

(d) $\beta \alpha^{n}(n=-3)$

Figura 3.3: Curvas representando classes de homotopia de $\pi_{1}\left(K^{2}\right)$.

Observação 3.1.11 (Veja [7, p. 139]) Seja $j$ um elemento de $\pi_{1}\left(K^{2}\right)$ e seja $J$ uma curva fechada simples representando $j$. Então $K^{2} \backslash J$ terá

(a) duas componentes, se $j=1$ (uma das quais é difeomorfa a um disco aberto);

(b) uma componente difeomorfa a um cilindro, se $j=\alpha^{ \pm 1}$; 
(c) uma componente difeomorfa a uma faixa de Möbius, se $j=\beta \alpha^{n}$ ou $j=\beta^{-1} \alpha^{n}$;

(d) duas componentes difeomorfas a uma faixa de Möbius, se $j=\beta^{ \pm 2}$.

Notemos que uma curva fechada simples na garrafa de Klein será de dois lados, se sua classe de homotopia for $\alpha^{ \pm 1}$ ou $\beta^{ \pm 2}$.

\subsection{O Teorema de Poincaré-Bendixson}

Nesta seção, desenvolvemos uma versão do Teorema de Poincaré-Bendixson para campos vetoriais contínuos na garrafa de Klein $K^{2}$.

Seja $M$ uma variedade compacta bidimensional e seja $\gamma$ uma trajetória de $X \in \mathfrak{X}(M)$. No que segue, $[p, q]_{\gamma}$ (respectivamente $\left.(p, q)_{\gamma}\right)$ denotará o arco fechado orientado (respectivamente o arco aberto orientado) de trajetória de $X$ contido em $\gamma$, começando em $p$ e terminando em $q$. A orientação deste arco é aquela induzida por $X$.

Seja $\Sigma$ um segmento transversal a $X$ e sejam $p, q \in \Sigma$. Denotaremos por $[p, q]_{\Sigma}$ o subintervalo de $\Sigma$ com extremos $p$ e $q$.

Para fluxos contínuos, o fato de que toda trajetória $\omega$-recorrente na garrafa de Klein $K^{2}$ é trivial foi provado em [9], usando-se o seguinte resultado de M. Peixoto [19]: Dada uma trajetória $\omega$-recorrente $\gamma$ de um fluxo contínuo, existe um círculo transversal por um ponto de $\gamma$.

Para tratarmos desta questão para campos vetoriais contínuos, introduziremos a noção de círculo semi-transversal e, em seguida, provaremos alguns resultados sobre existência de tais círculos em $K^{2}$.

Definição 3.2.1 Seja $\gamma$ uma trajetória de $X \in \mathfrak{X}(M)$ que intercepta um segmento transversal $\Sigma$ em pontos p e q. Diremos que a curva $[p, q]_{\gamma} \cup[p, q]_{\Sigma}$ formada pela união do arco de trajetória $[p, q]_{\gamma}$ de $X$ com o subintervalo $[p, q]_{\Sigma}$ de $\Sigma$ é um círculo semi-transversal a $X$ por $\gamma$, se ela for uma curva fechada simples de dois lados.

O próximo resultado estabelece condições para a existência de um círculo semi-transversal por uma trajetória injetiva de $X \in \mathfrak{X}\left(K^{2}\right)$. 
Lema 3.2.2 Seja $\gamma$ uma trajetória injetiva de $X \in \mathfrak{X}\left(K^{2}\right)$. Se $\gamma$ interceptar um segmento transversal $\Sigma$ em quatro pontos distintos, então existirá um círculo semi-transversal a $X$ por $\gamma$

\section{Demonstração:}

Sejam $p_{1}, p_{2}, p_{3}$ e $p_{4}$ pontos distintos e consecutivos da interseção de $\gamma \operatorname{com} \Sigma$. Sem perda de generalidade, podemos assumir que $p_{1}, p_{2}, p_{3}$ e $p_{4}$ formam uma seqüência monótona em $\Sigma$. Caso contrário, a construção de um círculo semi-transversal por $\gamma$ é imediata.

Consideremos, então, as seguintes curvas fechadas simples $\left[p_{1}, p_{2}\right]_{\gamma} \cup\left[p_{1}, p_{2}\right]_{\Sigma},\left[p_{2}, p_{3}\right]_{\gamma} \cup$ $\left[p_{2}, p_{3}\right]_{\Sigma}$ e $\left[p_{3}, p_{4}\right]_{\gamma} \cup\left[p_{3}, p_{4}\right]_{\Sigma}$ (veja Figura 3.4(a)). Fazendo uma pequena pertubação dessas curvas, obtemos curvas fechadas simples e disjuntas, $\widetilde{C}_{1}, \widetilde{C}_{2}$ e $\widetilde{C}_{3}$ (veja Figura $3.4(\mathrm{~b})$ ). Então, segue do Lema 3.1.10, que pelo menos uma dessas curvas é de dois lados. Logo, existe um círculo semi-transversal a $X$ por $\gamma$ e o lema está provado.

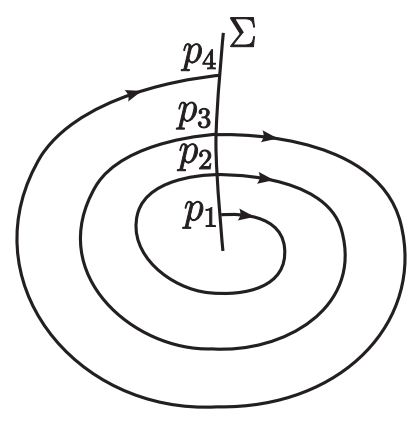

(a)

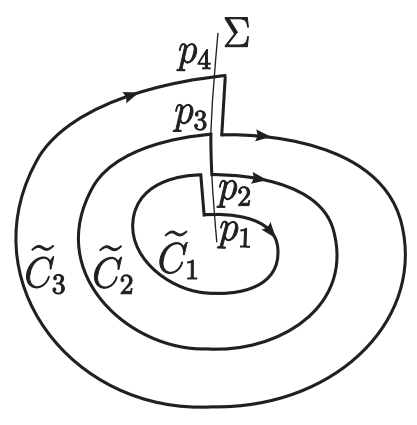

(b)

Figura 3.4: Curvas fechadas simples.

Corolário 3.2.3 Se $\gamma$ for uma trajetória injetiva fracamente $\omega$-recorrente (respectivamente fracamente $\alpha$-recorrente) de $X \in \mathfrak{X}\left(K^{2}\right)$, então existirá um círculo $C$ semi-transversal a $X$ por $\gamma$. Além disso, $K^{2} \backslash C$ será conexo.

\section{Demonstração:}

Suponhamos que $\gamma$ seja fracamente $\omega$-recorrente em $p \in \gamma$ e seja $\Sigma$ um segmento transversal passando por $p$. Como $p \in \omega(\gamma)$, então a semitrajetória positiva $\gamma_{p}^{+} \subset \gamma$ intercepta $\Sigma$ em um número infinitos de pontos. Daí, pelo Lema 3.2.2, existe um círculo $C=\left[p_{1}, p_{2}\right]_{\gamma} \cup\left[p_{1}, p_{2}\right]_{\Sigma}$ semi-transversal a $X$ por $\gamma$, onde $p_{1} \in \gamma_{p}^{+} \subset \gamma$ (veja Figura 3.5). 
Suponhamos que $p$ pertença à componente conexa de $\Sigma \backslash\left\{p_{2}\right\}$ que contém $p_{1}$ (veja as Figuras 3.5(a) e 3.5(b)). Vamos denotar por $p_{3}$ o primeiro ponto que a semitrajetória positiva $\gamma_{p_{2}}^{+} \subset \gamma$ intercepta essa componente. Como o arco de trajetória $\left(p_{2}, p_{3}\right)_{\gamma}$ é um caminho ligando os dois lados de $C$, sem interceptar $C$, então $K^{2} \backslash C$ é conexo.

No caso em que $p$ não pertence à componente conexa de $\Sigma \backslash\left\{p_{2}\right\}$ que contém $p_{1}$, também é verdade que $K^{2} \backslash C$ é conexo, pois o arco de trajetória $\left(p, p_{1}\right)_{\gamma}$ é um caminho ligando os dois lados de $C$ sem interceptar $C$ (veja Figura 3.5(c)). Assim, o corolário está provado.

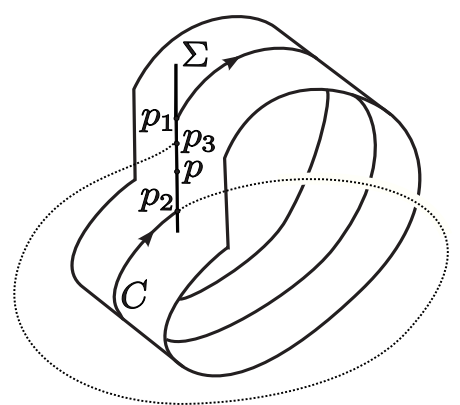

(a)

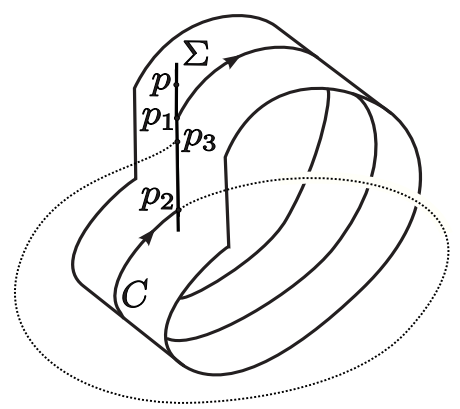

(b)

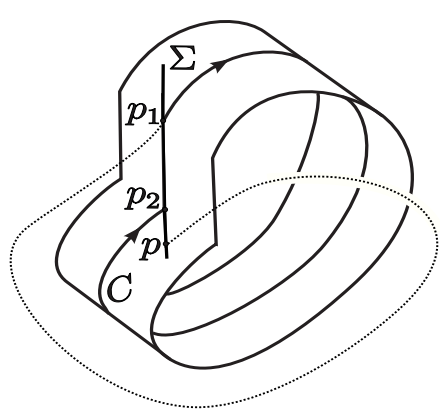

(c)

Figura 3.5: A conexidade de $K^{2} \backslash C$.

Agora, vamos estender o conceito de gráfico para campos vetoriais contínuos. Em seguida, mostraremos uma versão do Teorema de Poincaré-Bendixson o qual diz que, em qualquer campo vetorial contínuo na garrafa de Klein $K^{2}$, com um número finito de singularidades, o conjunto $\omega$-limite de uma trajetória injetiva fracamente $\omega$-recorrente é, necessariamente, uma trajetória periódica ou um gráfico.

Definição 3.2.4 Um gráfico para $X \in \mathfrak{X}(M)$ é um subconjunto fechado e conexo de $M$ constituído de finitas singularidades e de uma seqüência (finita ou infinita) de trajetórias injetivas e regulares tais que os conjuntos $\alpha$ e $\omega$-limites são singularidades.

Observação 3.2.5 Sejam $X \in \mathfrak{X}(M)$ e $N \subset M$ uma região homeomorfa a um cilindro. Suponhamos que o campo $X$ possua um número finito de singularidades em $N$. Se uma semitrajetória positiva injetiva $\gamma^{+}$estiver contida em $N$, então o seu conjunto $\omega$-limite apresentará as mesmas possibilidades que no Teorema de Poincaré-Bendixson em $\mathbb{R}^{2}$ (Teorema 1.3.3), ou seja, ou $\omega\left(\gamma^{+}\right)$será uma trajetória periódica ou será um ponto singular ou 
será um gráfico. A demonstração deste fato é similar àquela dada para $\mathbb{R}^{2}$, usando o fato que uma curva de Jordan $J$ em $N$ divide $N \backslash J$ em duas componentes conexas limitadas contendo, em seus bordos, a curva $J$.

Teorema 3.2.6 Seja $X \in \mathfrak{X}\left(K^{2}\right)$ um campo vetorial contínuo com um número finito de singularidades e seja $\gamma$ uma trajetória injetiva fracamente $\omega$-recorrente. Então $\omega(\gamma)$ é exatamente um dos seguintes conjuntos:

(i) Uma trajetória periódica;

(ii) Um gráfico.

\section{Demonstração:}

Pelo Corolário 3.2.3, existe um círculo $C$ semi-transversal a $X$ por $\gamma$ tal que $K^{2} \backslash C$ é conexo. Podemos assumir, pelo Lema 3.1.9, que $K^{2} \backslash C$ é um anel, onde os círculos fronteiras, que denotaremos por $C_{1}$ e $C_{2}$, são orientados pelas setas como na Figura 3.6. Tais círculos podem ser identificados por um homeomorfismo $h: C_{1} \rightarrow C_{2}$ que preserva orientação.

Afirmamos que existe $r \in \gamma$ tal que a semitrajetória positiva $\gamma_{r}^{+} \subset \gamma$ fica contida no anel $K^{2} \backslash C$ ou em uma faixa de Möbius. De fato, é suficiente mostrarmos que se $\gamma$ interceptar $C$ em três pontos consecutivos $p, q$ e $r$, então a semitrajetória positiva $\gamma_{r}^{+} \subset \gamma$ ficará contida em uma faixa de Möbius.

Denotemos por $\overline{p q}$ o arco de $C$ com extremos $p$ e $q$ contendo $r$. Como $K^{2} \backslash C$ é um anel e $\gamma$ é injetiva, a semitrajetória $\gamma_{r}^{+} \subset \gamma$ pode interceptar $C$ somente outra vez no arco $\overline{r q} \subset \overline{p q}$ com extremos $r$ e $q$ (veja Figura 3.6). Portanto a semitrajetória $\gamma_{r}^{+} \subset \gamma$ fica contida na faixa de Möbius, representada pelo diagrama da Figura 3.7. Isto completa a prova da nossa afirmação.

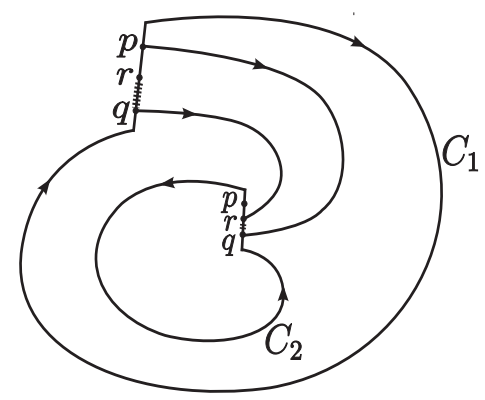

Figura 3.6: Anel com fronteira $C_{1}$ e $C_{2}$.

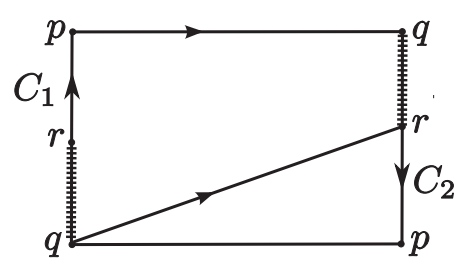

Figura 3.7: Faixa de Möbius. 
Agora, usando o fato de que o cilindro é um recobrimento duplo orientado da faixa de Möbius e usando a Observação 3.2.5, concluímos que $\omega(\gamma)$ é uma trajetória periódica ou um gráfico. Assim, terminamos a demonstração do teorema.

Como conseqüência imediata da demonstração do Teorema 3.2.6, obtemos uma versão do Teorema 2 de [9] (veja também [1] e [15]) para campos vetoriais contínuos. Apresentamos este resultado no corolário seguinte.

Corolário 3.2.7 Um campo vetorial contínuo $X \in \mathfrak{X}\left(K^{2}\right)$ não possui trajetória injetiva $\omega$-recorrente.

Agora, prosseguimos para apresentar uma versão do Teorema de Poincaré-Bendixson na garrafa de Klein $K^{2}$ no caso em que a trajetória injetiva de $X \in \mathfrak{X}\left(K^{2}\right)$ não é fracamente $\omega$-recorrente. Os próximos dois resultados facilitarão a demonstração de tal teorema.

Proposição 3.2.8 Seja $X \in \mathfrak{X}(M)$ um campo vetorial contínuo em uma variedade compacta orientável bidimensional $M$ e seja $\gamma$ uma trajetória injetiva que não é fracamente $\omega$-recorrente. Se $\widetilde{\gamma}$ for uma trajetória periódica acompanhada por $\gamma$, então $\omega(\gamma)=\widetilde{\gamma}$.

\section{Demonstração:}

As idéias principais desta prova foram baseadas em [2, Lema 1.6, p. 49].

Como $M$ é orientável, então $\widetilde{\gamma}$ tem uma vizinhança $U$ homeomorfa a um anel tal que $\widetilde{\gamma}$ separa $U$ em duas componentes conexas. Então, segue da continuidade de $X$, que o conjunto dos pontos singulares de $X$ é fechado e, portanto, disjunto de $\widetilde{\gamma}$. Assim, podemos considerar que a vizinhança $U$ seja suficientemente pequena de tal forma que ela não contenha pontos singulares.

Seja $\Sigma$ um segmento transversal passando por um ponto $p \in \widetilde{\gamma}$ tal que $\Sigma \subset U$ e $\widetilde{\gamma}$ intercepta $\Sigma$ somente em $p$. Como $p \in \omega(\gamma)$, então $\gamma$ intercepta $\Sigma$ infinitas vezes. Sejam $p_{1}$ e $p_{2}$ pontos consecutivos de interseção de $\gamma$ e $\Sigma$ com respeito ao tempo crescente (veja Figura 3.8). Uma vez que $\widetilde{\gamma}$ é acompanhada por $\gamma$, podemos tomar $p_{1}$ suficientemente próximo de $p$ para que o arco de trajetória $\left[p_{1}, p_{2}\right]_{\gamma}$ fique contido em $U$. Como $\widetilde{\gamma}$ separa $U$ e $\widetilde{\gamma} \cap \gamma=\varnothing$, então $\left[p_{1}, p_{2}\right]_{\gamma}$ pertence a uma única componente de $U \backslash \widetilde{\gamma}$. Portanto a curva fechada simples $\Gamma=\left[p_{1}, p_{2}\right]_{\gamma} \cup\left[p_{1}, p_{2}\right]_{\Sigma}$ não intercepta $\widetilde{\gamma}$ e está contida em $U$. 
Se $\Gamma$ limitar um domínio simplesmente conexo $D$ em $U$, então $D \subset U$ conterá um ponto singular. Este fato segue dos Teoremas 1.3.11 e 1.3.12. Mas isso contradiz a escolha da vizinhança $U$. Portanto a curva $\Gamma$ não é homotópica a um ponto em $U$ e, junto com $\widetilde{\gamma}$, limitam um domínio anular $K \subset U$ (veja Figura 3.8).

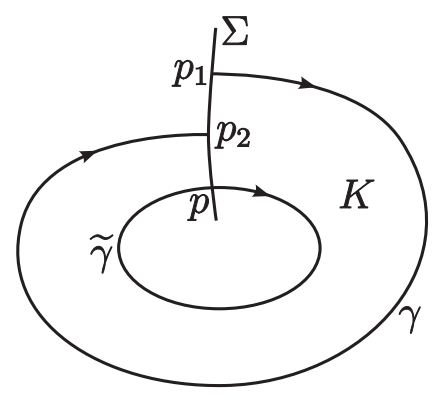

Figura 3.8: $\mathrm{O}$ anel $K$.

Denotemos por $\Sigma_{R}$ e $\Sigma_{L}$ as componentes conexas de $\Sigma \backslash\{p\}$. Para fixar as idéias, suponhamos que $p_{1}, p_{2} \in \Sigma_{R}$. Como $p$ pertence ao conjunto $\omega$-limite de $\gamma$, uma, e somente uma, das seguintes alternativas ocorre:

(a) a semitrajetória positiva $\gamma_{p_{2}}^{+} \subset \gamma$ não intercepta $\Sigma_{L}$;

(b) $\gamma_{p_{2}}^{+} \subset \gamma$ intercepta $\Sigma_{L}$ em pontos arbitrariamente próximos de $p$.

Façamos algumas observações com respeito ao caso $(a)$. Mostraremos que $p_{2}$ está em $\Sigma$ entre $p_{1}$ e $p$. Suponhamos que isto não ocorra, isto é, suponhamos que $p_{1}$ esteja em $\Sigma$ entre $p_{2}$ e $p$. Então $\gamma_{p_{2}}^{+} \subset \gamma$ deixa o anel $K$ e não o intercepta mais, pois em $\left[p_{1}, p_{2}\right]_{\Sigma}$ todas as semitrajetórias saem de $K$ e $\gamma$ é injetiva (veja Figura 3.9). Mas, neste caso (a), isso significa que $\gamma_{p_{2}}^{+} \subset \gamma$ não intercepta $\Sigma$ em qualquer vizinhança de $p$, contradizendo $p \in \omega(\gamma)$.

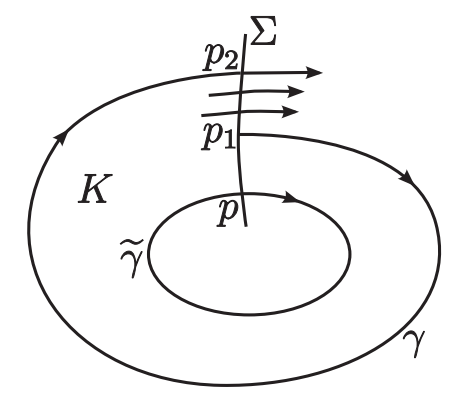

Figura 3.9: $p_{1}$ entre $p_{2}$ e $p$. 
Agora, provemos o teorema no caso $(a)$. Pelas observações feitas no parágrafo anterior, sabemos que $p_{2}$ está em $\Sigma$, entre $p_{1}$ e $p$. Denotemos por $p_{3}$ o primeiro ponto onde a semitrajetória positiva $\gamma_{p_{2}}^{+} \subset \gamma$ intercepta $\Sigma$. Afirmamos que $p_{3}$ está em $\Sigma$, entre $p_{2}$ e $p$. De fato, a semitrajetória $\gamma_{p_{2}}^{+} \subset \gamma$, ao entrar em $K$, não pode deixá-lo, pois em $\left[p_{1}, p_{2}\right]_{\Sigma}$ todas as semitrajetórias entram em $K$ e $\gamma$ é injetiva (veja Figura 3.8). Por essa razão, $p_{3} \notin\left[p_{1}, p_{2}\right]_{\Sigma}$ e, portanto, $p_{3} \in\left[p_{2}, p\right]_{\Sigma}$. Prosseguindo dessa forma, obtemos uma seqüência $\left(p_{n}\right)_{n \in \mathbb{N}}$ em $\Sigma$ com as seguintes propriedades:

$\left(a_{1}\right) p_{n+1} \in \gamma_{p_{n}}^{+} \cap \Sigma$, onde $\gamma_{p_{n}}^{+} \subset \gamma$;

$\left(a_{2}\right)$ o ponto $p_{n+1}$ está em $\Sigma$, entre $p_{n}$ e $p$ (monotonicidade);

$\left(a_{3}\right) \gamma_{p_{1}}^{+} \subset \gamma$ não intercepta $\Sigma$ em qualquer ponto, exceto em $p_{n}, n \in \mathbb{N}$ (veja Figura 3.10).

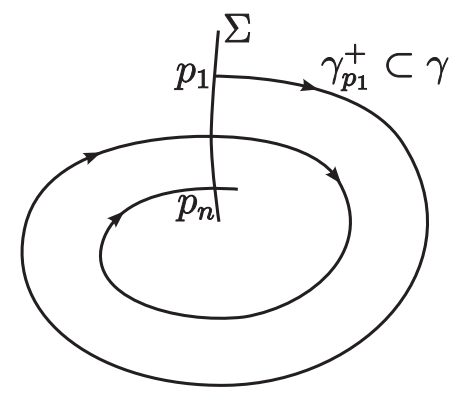

Figura 3.10: A seqüência monótona $\left(p_{n}\right)_{n \in \mathbb{N}}$.

Segue da propriedade $\left(a_{2}\right)$ que a seqüência $\left(p_{n}\right)_{n \in \mathbb{N}}$ tem um ponto de acumulação $q \in \Sigma$. Como $p \in \omega(\gamma)$, então $q=p$. Além disso, $\left(a_{2}\right)$ e $\left(a_{3}\right)$ implicam que $\omega(\gamma) \cap \Sigma=\{p\}$.

Uma vez que a semitrajetória $\gamma_{p_{2}}^{+} \subset \gamma$ está contida em $K$, o mesmo raciocínio utilizado anteriormente nos permite concluir que:

$\left(a_{4}\right)$ qualquer segmento transversal passando por um ponto $\widetilde{p} \in \widetilde{\gamma}$ intercepta $\omega(\gamma)$ somente em $\widetilde{p}$.

De posse desse resultado, suponhamos que $\omega(\gamma) \backslash \widetilde{\gamma} \neq \varnothing$. Então $\omega(\gamma) \backslash \widetilde{\gamma}$ tem um ponto de acumulação $\widetilde{p} \in \widetilde{\gamma}$, pois $\omega(\gamma)$ é conexo. Seja $\Sigma_{\widetilde{p}}$ um segmento transversal passando por $\widetilde{p}$. Usando a Proposição 2.2 .3 e o fato de que toda vizinhança de $\widetilde{p}$ contém pontos de $\omega(\gamma) \backslash \widetilde{\gamma}$, então existe uma trajetória $\gamma_{p_{*}} \subset \omega(\gamma) \subset \bar{K}$ passando por um ponto $p_{*} \in \omega(\gamma) \backslash \widetilde{\gamma}$ e interceptando $\Sigma_{\widetilde{p}}$. O ponto de interseção é necessariamente $\widetilde{p}$, devido a $\left(a_{4}\right)$. 
Suponhamos que $\gamma_{p_{*}}(\tau) \in \widetilde{\gamma}$, quando $\gamma_{p_{*}}(t) \notin \widetilde{\gamma}$, para $t$ entre 0 e $\tau$. Consideremos um segmento transversal $\Sigma_{*}$ passando por $\gamma_{p_{*}}(\tau)$. Então uma pequena translação de $\Sigma_{*}$, em uma direção conveniente, é um segmento transversal que intercepta $\widetilde{\gamma}$ e $\gamma_{p_{*}}$ em dois pontos distintos (veja Figura 3.11). Esta contradição com $\left(a_{4}\right)$ demonstra o teorema no caso $(a)$.

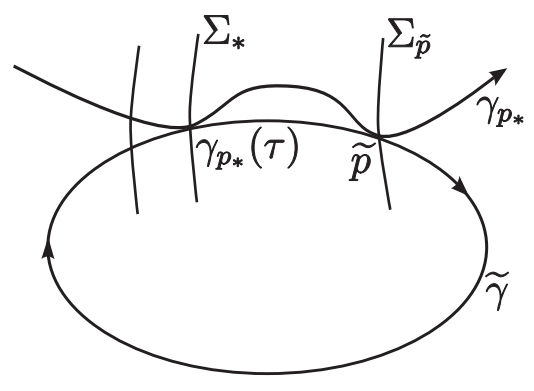

Figura 3.11: Os segmentos transversais $\Sigma_{\widetilde{p}}$ e $\Sigma_{*}$.

Consideremos, agora, o caso (b). Segue dos argumentos precedentes que, neste caso, a semitrajetória $\gamma_{p_{2}}^{+} \subset \gamma$ deixa $K$ e não o intercepta mais. Analogamente à construção de $K$, construímos um anel $\check{K} \subset U$ limitado por $\widetilde{\gamma}$ e por uma curva fechada simples formada pela união de um arco $\left[\check{p}_{1}, \check{p}_{2}\right]_{\gamma}$ da semitrajetória $\gamma_{p_{2}}^{+} \subset \gamma$ com o subintervalo $\left[\check{p}_{1}, \check{p}_{2}\right]_{\Sigma}$ de $\Sigma$ (veja Figura 3.12). Como $\widetilde{\gamma} \subset \omega(\gamma)$, então a semitrajetória $\gamma_{p_{2}}^{+} \subset \gamma$ tem que entrar no anel $\check{K}$ e, portanto, $\check{p}_{2}$ está entre $\check{p}_{1}$ e $p$ necessariamente.

O restante da prova é inteiramente análogo ao caso $(a)$, com $K, p_{1}$ e $p_{2}$ substituídos por $\check{K}, \check{p}_{1}$ e $\check{p}_{2}$ respectivamente.

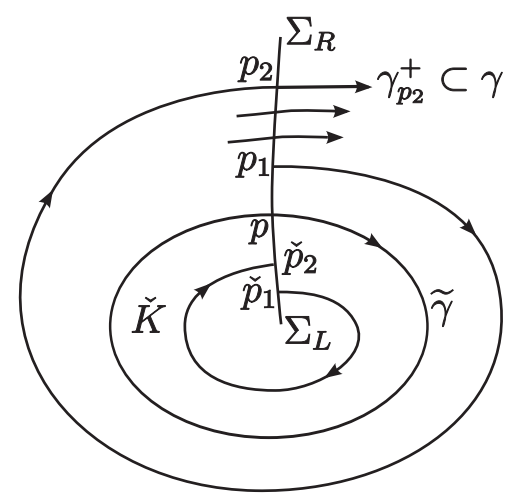

Figura 3.12: O anel $\check{K}$.

Proposição 3.2.9 Sejam $X \in \mathfrak{X}\left(K^{2}\right)$ e $\gamma$ uma trajetória injetiva que não é fracamente $\omega$ recorrente. Suponhamos que o campo vetorial $X$ possua um número finito de singularidades 
em $\omega(\gamma)$. Se $\widetilde{\gamma}$ for uma trajetória injetiva acompanhada por $\gamma$, então $\omega(\widetilde{\gamma})$ e $\alpha(\widetilde{\gamma})$ serão singularidades.

\section{Demonstração:}

Suponhamos que $\omega(\widetilde{\gamma})$ contenha um ponto regular $p$. Seja $\Sigma$ um segmento transversal passando por $p$. Como $p \in \omega(\widetilde{\gamma})$, então a semitrajetória positiva $\widetilde{\gamma}^{+} \subset \widetilde{\gamma}$ intercepta $\Sigma$ em um número infinitos de pontos. Daí, pelo Lema 3.2.2, existe um círculo semi-transversal a $X$ por $\widetilde{\gamma}$, digamos $\widetilde{C}=\left[p_{1}, p_{2}\right]_{\widetilde{\gamma}} \cup\left[p_{1}, p_{2}\right]_{\Sigma}$. Sendo $\widetilde{\gamma}$ acompanhada por $\gamma$, podemos construir um círculo $C$ semi-transversal a $X$ por $\gamma$, suficientemente próximo de $\widetilde{C}$, com $p_{1}$ ou $p_{2}$ pertencente a $C$ (veja Figura 3.13).

Para fixar as idéias, suponhamos que $p_{1}$ pertença a $C$ (Figura 3.13(a)). Como $p_{1} \in \omega(\gamma)$, existem arcos de trajetória de $X$ contidos em $\gamma$, ligando os dois lados de $C$ e sem interceptar $C$. Logo $K^{2} \backslash C$ é conexo.

Agora, o mesmo raciocínio que utilizamos para demonstrar o Teorema 3.2.6 nos permite concluir que $\omega(\gamma)$ é um gráfico, pois $\widetilde{\gamma}$ é uma trajetória não periódica contida em $\omega(\gamma)$. Mas isso contradiz a existência de um ponto regular $p$ em $\omega(\widetilde{\gamma})$. Logo, $\omega(\widetilde{\gamma})$ é um ponto singular.

Analogamente, mostra-se que $\alpha(\widetilde{\gamma})$ é um ponto singular pertencente a $\omega(\gamma)$.

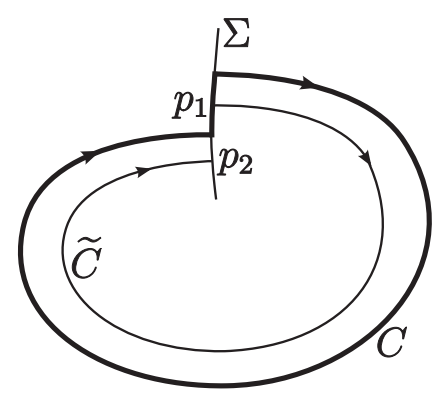

(a) $p_{1} \in C$.

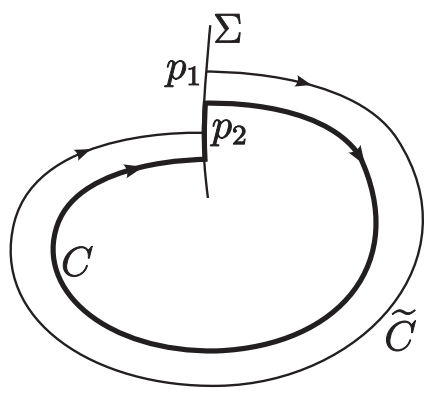

(b) $p_{2} \in C$.

Figura 3.13: Os círculos semi-transversais $C$ e $\widetilde{C}$.

Teorema 3.2.10 Seja $X \in \mathfrak{X}\left(K^{2}\right)$ um campo vetorial contínuo com um número finito de singularidades. Se $\gamma$ for uma trajetória injetiva não fracamente $\omega$-recorrente, então $\omega(\gamma)$ será exatamente um dos seguintes conjuntos:

(i) Uma singularidade;

(ii) Uma trajetória periódica; 
(iii) Um gráfico.

\section{Demonstração:}

Se $\omega(\gamma)$ não contiver pontos regulares, então $\omega(\gamma)$ será uma única singularidade, pois $X$ tem um número finito de singularidades e $\omega(\gamma)$ é conexo.

Para prosseguirmos com a demonstração, podemos supor que $\omega(\gamma)$ contém um ponto regular $p$. Consideremos um segmento $\Sigma$ transversal a $X$ por $p$. Pelo Lema 3.2.2, existe um círculo semi-transversal a $X$ por $\gamma$, digamos $C=\left[p_{1}, p_{2}\right]_{\gamma} \cup\left[p_{1}, p_{2}\right]_{\Sigma}$.

Se $C$ for uma curva não homotópica a um ponto em $K^{2}$, sabemos que $K^{2} \backslash C$ será um cilindro (se $K^{2} \backslash C$ for conexo, pelo Lema 3.1.9) ou consistirá de duas faixas de Möbius (veja Observação 3.1.11). Assim, existe $r \in \gamma$ tal que a semitrajetória positiva $\gamma_{r}^{+} \subset \gamma$ fica contida no cilindro ou em uma faixa de Möbius. Portanto, $\omega(\gamma)$ é uma trajetória periódica ou um gráfico.

Se $C$ for uma curva homotópica a um ponto em $K^{2}$, então $C$ limitará um disco aberto $D$ em $K^{2}$. Notemos que, neste caso, $p \notin C$. Se $p \in D$, então, segue do Teorema 1.3.3, que $\omega(\gamma)$ será uma trajetória periódica ou um gráfico.

No que segue, podemos supor que todos os círculos semi-transversais a $X$ por $\gamma$ são homotópicos a um ponto em $K^{2}$ e que $p$ não pertence aos discos abertos que estes círculos limitam. Assim, $p \notin D$ (veja Figura 3.14).

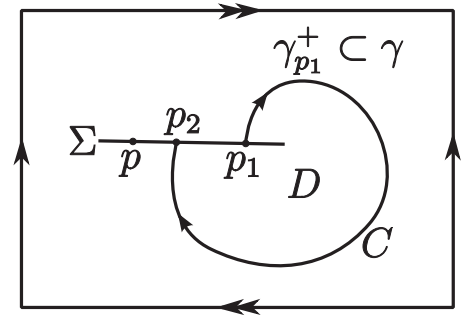

Figura 3.14: O círculo $C$ homotópico a um ponto em $K^{2}$.

Como $p \in \omega(\gamma)$, então a semitrajetória positiva $\gamma_{p_{1}}^{+} \subset \gamma$ intercepta $\Sigma$ segundo uma seqüência $\left(p_{n}\right)_{n \in \mathbb{N}}$, onde $p_{n}=\gamma\left(t_{n}\right),\left(t_{n}\right)_{n \in \mathbb{N}}$ é uma seqüência crescente de números reais e $p_{n} \rightarrow p$ quando $n \rightarrow \infty$.

Consideremos as curvas fechadas

$$
C_{n}=\left[p_{n}, p_{n+1}\right]_{\gamma} \cup\left[p_{n}, p_{n+1}\right]_{\Sigma}, n=1,2, \ldots
$$


Afirmamos que $C_{n}$ é uma curva de dois lados para todo $n \in \mathbb{N}$. De fato, suponhamos, por contradição, que exista $n_{0} \geq 2$ tal que $C_{n_{0}}$ é uma curva de um lado. Então temos as seguintes possibilidades:

(a) existe $n>n_{0}$ tal que $C_{n}$ é uma curva de dois lados;

(b) $C_{n}$ é uma curva de um lado para todo $n \geq n_{0}$.

O caso $(a)$ não pode ocorrer, pois se ocorrer, $C_{n}$ será um círculo semi-transversal não homotópico a um ponto em $K^{2}$, o que contradiz a nossa suposição.

Mostraremos que $(b)$ também não é verificado. Se $\left(p_{n}\right)_{n \in \mathbb{N}}$ não for uma seqüência monótona em $\Sigma$, então $\gamma_{p_{1}}^{+} \subset \gamma$ interceptará $\Sigma$ em três pontos não consecutivos. Daí poderemos construir um círculo semi-transversal a $X$ por $\gamma$ passando por esses pontos e não homotópico a um ponto em $K^{2}$, o que contradiz nossa suposição. Portanto $\left(p_{n}\right)_{n \in \mathbb{N}}$ é uma seqüência necessariamente monótona. Por outro lado, pelo Lema 3.1.10, só podem haver duas curvas disjuntas de um lado na garrafa de Klein $K^{2}$. Logo, não existe $n_{0} \geq 2$ tal que $C_{n_{0}}$ é de um lado. Assim (b) também não vale.

Segue da afirmação demonstrada e da hipótese de que todos os círculos semi-transversais a $X$ por $\gamma$ são homotópicos a um ponto em $K^{2}$ que $\left(p_{n}\right)_{n \in \mathbb{N}}$ é uma seqüência monótona em $\Sigma$. Conseqüentemente, $\omega(\gamma) \cap \Sigma=p$. Pela arbitrariedade do ponto $p \in \omega(\gamma)$, isto implica que existe uma única trajetória de $X$ passando por $p$ e contida em $\omega(\gamma)$, pois caso contrário, existiriam dois pontos distintos de $\omega(\gamma)$ em um mesmo segmento transversal e, conseqüentemente, conseguiríamos construir um círculo semi-transversal a $X$ por $\gamma$ não homotópico a um ponto em $K^{2}$.

Finalmente, se $\omega(\gamma)$ contiver uma trajetória periódica, segue da Proposição 3.2.8 e do fato que o toro é um recobrimento duplo orientado da garrafa de Klein, que $\omega(\gamma)$ será uma trajetória periódica. Suponhamos que $\omega(\gamma)$ não contenha qualquer trajetória periódica. Então todas as trajetórias de $X$ em $\omega(\gamma)$ são injetivas. Daí, pela Proposição $3.2 .9, \omega(\gamma)$ é um gráfico e o teorema está demonstrado. 



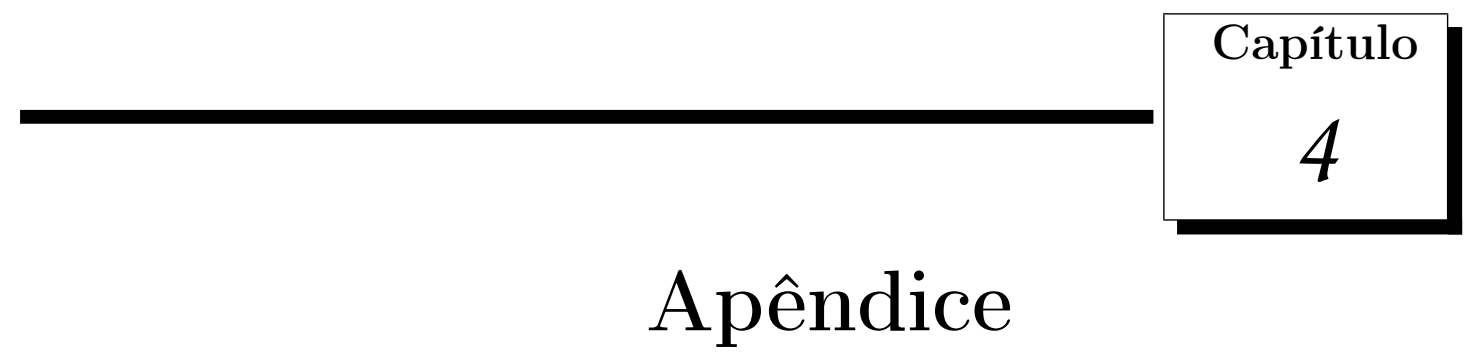

Neste apêndice, vamos considerar $M$ uma variedade compacta bidimensional de classe $C^{\infty}$ e $X \in \mathfrak{X}(M)$ um campo vetorial contínuo. Denotemos por $F$ o conjunto dos pontos singulares de $X$, onde $F$ é um conjunto finito ou infinito.

Proposição 4.0.11 Nas condições acima, existe um campo vetorial contínuo $Y \in \mathfrak{X}(M)$ cujo o conjunto de pontos singulares é precisamente $F$ e tal que:

(i) $Y$ tem o mesmo retrato de fase de $X$;

(ii) todas as trajetórias de $Y$ são regulares, a menos das trajetórias dadas por pontos singulares.

\section{Demonstração:}

Usando a Proposição 3.2 de [8], existe uma família $\left\{M_{n}\right\}_{n \in \mathbb{N}}$ de subconjuntos compactos de $M$ tal que $\bigcap_{n=1}^{\infty} M_{n}=F$, onde $M_{1}=M$ e $M_{n+1} \subset \operatorname{Int}\left(M_{n}\right)$, para todo $n \in \mathbb{N}$. Definamos $V_{n}=\operatorname{Int}\left(M_{n}\right) \backslash M_{n+2}, n \in \mathbb{N}$. Certamente $\left\{V_{n}\right\}_{n \in \mathbb{N}}$ é uma cobertura aberta localmente finita de $M \backslash F$.

Seja $\left\{\psi_{n}: M \backslash F \rightarrow[0,1]: n \in \mathbb{N}\right\}$ uma partição da unidade estritamente subordinada a esta cobertura. Para cada $n \in \mathbb{N}$, estendemos $\psi_{n}$ a uma aplicação contínua $\widetilde{\psi}_{n}$ em $M$, identificando $\widetilde{\psi}_{n}(p)=0$ para todo $p \in F$. Assim, $\widetilde{\psi}_{n} X \in \mathfrak{X}(M)$, para cada $n \in \mathbb{N}$.

Sejam $\rho_{0}=1, \rho_{1}=1$ e $\rho_{n}=d\left(M_{n+2}, M \backslash M_{n}\right):=\inf \left\{d(p, q): p \in M_{n+2}, q \in M \backslash M_{n}\right\}$, para $n=2,3, \ldots$, onde $d(.,$.$) designa uma métrica em M$. Denotemos por $\|\cdot\|$ a norma 
dada por esta métrica. Para cada $n \in \mathbb{N}$, escolhemos um número real positivo $c_{n}$ tal que

$$
\left\|c_{n} \widetilde{\psi}_{n} X\right\|<\min \left\{\frac{1}{2^{n}}, \frac{\rho_{n-1}}{3}, \frac{\rho_{n}}{3}, \frac{\rho_{n+1}}{3}\right\}
$$

Como

$$
\sum_{n=1}^{\infty}\left\|c_{n} \widetilde{\psi}_{n} X\right\| \leq \sum_{n=1}^{\infty} \frac{1}{2^{n}}<\infty
$$

então a série $\sum_{n=1}^{\infty} c_{n} \widetilde{\psi}_{n} X$ converge para um campo vetorial contínuo $Y \in \mathfrak{X}(M)$ como requerido em $(i)$.

Resta mostrarmos que $Y$ satisfaz $(i i)$. Para cada $n=2,3, \ldots$, vale

$$
\begin{aligned}
\left\|\left.\left(\sum_{n=1}^{\infty} c_{n} \widetilde{\psi}_{n} X\right)\right|_{V_{n}}\right\| & =\left\|\left.\left(c_{n-1} \widetilde{\psi}_{n-1} X+c_{n} \widetilde{\psi}_{n} X+c_{n+1} \widetilde{\psi}_{n+1} X\right)\right|_{V_{n}}\right\| \\
& \leq\left\|c_{n-1} \widetilde{\psi}_{n-1} X\right\|+\left\|c_{n} \widetilde{\psi}_{n} X\right\|+\left\|c_{n+1} \widetilde{\psi}_{n+1} X\right\| \\
& \leq \rho_{n} .
\end{aligned}
$$

Isto implica que $\left\|\left.Y\right|_{V_{n}}\right\| \leq \rho_{n}$.

Seja $\gamma$ uma trajetória de $Y$ tal que $\gamma(0) \in \overline{M \backslash M_{n}}$ e $\gamma(\tau) \in M_{n+2}$, onde $\tau>0$ e $n=2,3, \ldots$ Então

$$
\rho_{n} \leq\|\gamma(\tau)-\gamma(0)\|=\left\|\int_{0}^{\tau} \gamma^{\prime}(s) d s\right\|=\left\|\int_{0}^{\tau} Y(\gamma(s)) d s\right\| \leq \tau \rho_{n}
$$

Logo $\tau \geq 1$, sempre que $\gamma(0) \in \overline{M \backslash M_{n}}$ e $\gamma(\tau) \in M_{n+2}$, para cada $n=2,3, \ldots$. Portanto, concluímos que $\gamma$ pode atingir uma singularidade somente em tempo infinito pela definição de $F$, pois $M_{n+1} \subset \operatorname{Int}\left(M_{n}\right)$, para todo $n \in \mathbb{N}$. Isto demonstra (ii). 


\section{Referências Bibliográficas}

[1] S. Kh. Aranson, Trajectories on two-dimensional nonorientable manifolds, Mat. Sb. 80 (1969), 314-333.

[2] S. Kh. Aranson, G. R. Belitsky e E. V. Zhuzhoma, Introduction to the Qualitative Theory of Dynamical Systems on Surfaces, AMS, Math. Monogr. volume 153, 1996.

[3] I. Bendixson, Sur les courbes définies par des équations différentielles, Acta Math. 24 (1901), 1-88.

[4] E. M. Bonotto e M. Federson, Limit sets and the Poincaré-Bendixson Theorem in impulsive semidynamical systems, J. Diff. Equations 244 (2008), 2334-2349.

[5] K. Ciesielski, The Poincaré-Bendixson theorems for two-dimensional semiflows, Topological Methods in Nonlinear Analysis 3 (1994), 163-178.

[6] D. P. Demuner, M. Federson e C. Gutierrez, The Poincaré-Bendixson Theorem on the Klein bottle for continuous vector fields, Discrete and Continuous Dynamical Systems, aceito para publicação.

[7] C. Godbillon, Dynamical Systems on Surfaces, Springer-Verlag, New York, 1983.

[8] C. Gutierrez, Smoothing continuous flows on two-manifolds and recurrences, Ergodic Theory Dynam. Systems 6 (1986), 17-44.

[9] C. Gutierrez, Structural stability for flows on the torus with a cross-cap, Trans. Amer. Math. Soc. 241 (1978), 311-320. 
[10] F. Haas, Poincaré-Bendixson type theorems for two-dimensional manifolds different from the torus, Ann. of Math. 59 (1954), 292-299.

[11] O. Hajek, Dynamical Systems in the Plane, Academic Press, 1968.

[12] P. Hartman, Ordinary Differential Equations, John Wiley and Sons. Inc., 1964.

[13] H. Kneser, Reguläre Kurvenscharen auf Ringflächen, Math. Ann. 91 (1924), 135-154.

[14] E. L. Lima, Grupo Fundamental e Espaços de Recobrimento, Projeto Euclides, IMPA, 2006.

[15] N. G. Markley, The Poincaré-Bendixson theorem for the Klein bottle, Trans. Amer. Math. Soc. 135 (1969), 159-165.

[16] W. S. Massey, Algebraic Topology: an Introduction, Harcourt-Brace-World, 1967.

[17] M. A. Newman, Elements of the Topology of Plane Sets of Points, Cambridge University Press (VII 4), 1954.

[18] J. Palis Jr. e W. Melo, Introdução aos Sistemas Dinâmicos, Projeto Euclides, IMPA, 1978.

[19] M. M. Peixoto, Structural stability on two-dimensional manifolds, Topology 1 (1962), 101-120.

[20] H. Poincaré, Sur les courbes définies par les équations différentielles, J. Math. Pures Appl. 2 (1886), 151-217.

[21] H. Poincaré, Sur les courbes définies par les équations différentielles, Oeuvres, vol I, Gauthier Villars, Paris 1928, pp. 3-84, 90-161, 167-221.

[22] T. Radó, Über den Begriff der Riemannschen Fläche, Acta Litt. Sci. Szeged. 2 (1925), 101-121.

[23] A. J. Schwartz, A generalization of a Poincaré-Bendixson theorem to closed twodimensional manifolds, Amer. J. Math. 85 (1963), 453-458.

[24] E. Spanier, Algebraic Topology, Springer-Verlag, New York, 1966. 